\title{
A posteriori error analysis for Schwarz overlapping domain decomposition methods
}

\author{
Jehanzeb Chaudhry, Don Estep and Simon Tavener
}

\begin{abstract}
Domain decomposition methods are widely used for the numerical solution of partial differential equations on high performance computers. We develop an adjoint-based a posteriori error analysis for both multiplicative and additive overlapping Schwarz domain decomposition methods. The numerical error in a user-specified functional of the solution (quantity of interest) is decomposed into contributions that arise as a result of the finite iteration between the subdomains and from the spatial discretization. The spatial discretization contribution is further decomposed into contributions arising from each subdomain. This decomposition of the numerical error is used to construct a two stage solution strategy that efficiently reduces the error in the quantity of interest by adjusting the relative contributions to the error.
\end{abstract}

Keywords - Schwarz overlapping domain decomposition, a posteriori error estimation, adaptive computation

\section{Introduction}

We derive and implement an adjoint-based a posteriori error analysis for overlapping domain decomposition methods for elliptic boundary value problems, examining both additive and multiplicative Schwarz algorithms. Domain decomposition methods (DDMs) arrive at the solution of a problem defined over a domain by combining the solutions of related problems posed on subdomains. The problems posed on subdomains often require less computational resources and some of the first uses of DDMs for practical applications were in low-memory or limited computation scenarios 28, 33. Recently DDMs have seen increased use in the context of distributed and parallel computing [27. 32, 34, 35, 36]. In this article, we follow the presentation in [32.

In overlapping DDMs, each subdomain has a non-empty intersection with at least one other subdomain and typically state information is exchanged between the subdomains. The theoretical properties of the multiplicative Schwarz method and some of its variants were studied in 30. The variant of this method suitable for parallel computing, called the additive Schwarz method, was introduced in [18. Non-overlapping DDMs, in which the subdomains have empty intersection and state and derivative information is exchanged through their common interfaces, is an alternative approach [29].

Adjoint-based a posteriori error analysis for systems of ordinary and partial differential equations has an extensive history [3, 4, 19, 20, 24, 25], and has been applied to a wide range of applications and numerical methods 1, 2, 7, 11, 13, 14, 9, 10, 15, 17, 16, 26. Adjoint-based a posteriori error analysis classically considers a differential equation

$$
L(u(\mathbf{x}, t))=g(\mathbf{x}, t),
$$

where $L$ denotes the differential operator, and a Quantity of Interest (QoI), expressed as a linear functional

$$
Q(u)=(u, \psi),
$$

where $(\cdot, \cdot)$ denotes the $L_{2}$ inner product and the function $\psi$ is chosen to yield the desired information. Given the numerical approximation $U$ to the analytical solution, the residual $R(U)=g-L(U)$ quantifies the effects of discretization on the evaluation of the differential equation, but it does not provide the error in the QoI, $(u-U, \psi)$. The relation between the residual and the error is derived from solving an adjoint problem.

For linear problems, the adjoint operator $\mathcal{L}^{*}: Y^{*} \rightarrow X^{*}$ of a linear operator $\mathcal{L}: X \rightarrow Y$ between Banach spaces $X, Y$ with dual spaces $X^{*}, Y^{*}$ is defined by the bilinear identity

$$
\left\langle\mathcal{L} x, y^{*}\right\rangle_{Y}=\left\langle x, \mathcal{L}^{*} y^{*}\right\rangle_{X}, \quad x \in X, y^{*} \in Y^{*},
$$

where $\langle\cdot, \cdot\rangle_{S}$ denotes duality-pairing in the space $S \in\{X, Y\}$. The adjoint problem associated with (1) is

$$
L^{*} \phi=\psi \text {. }
$$


This yields the error estimate,

$$
\text { Error in the QoI }=(u-U, \psi)=(R(U), \phi) .
$$

We estimate the numerical error in the quantity of interest by numerically solving the adjoint problem (4), computing the residual, and evaluating (5).

Classical a posteriori error analysis for the numerical solution of differential equations assumes the use of fully implicit discretization methods in which the approximate solution is computed exactly for which the adjoint of the forward operator (4) produces a useful adjoint solution. The adjoint of the discrete solution operator when implementing more complex, multistage solution methods is much more complicated to define. If the steps in the solution process are written as compositions of operators, then the appropriate adjoint can typically be written as a composition of adjoints associated with various steps of discretization. The resulting error estimate must then use the appropriate adjoint to weight specific residuals and include additional terms quantifying the difference between this adjoint and the adjoint of the overall problem (4). The correct choice of adjoint and residuals also enables a decomposition of the total error into distinct sources of error, such as discretization, iteration, transfer, projection and quadrature errors. These concepts are illustrated in an analysis of iterative solvers for non-autonomous evolution problems in [8, in an analysis of a multirate iterative solver for ordinary differential equations in [22, and in an analysis of an iterative multi-discretization method for reaction-diffusion systems in [12]. An a posteriori error analysis for non-overlapping DDM is carried out in [7. To the best of our knowledge, an a posteriori error analysis for overlapping DDMs has not been performed.

Adjoint-based a posteriori error estimates can provide useful information for designing efficient adaptive solution strategies. During the first "pre-processing" step (stage 1), a solution is computed on a relatively coarse discretization together with an accurate a posteriori error estimate that quantifies the contributions of all sources of error. The information provided by the first stage is used to guide discretization choices for a "production level" (stage 2) computation. This strategy is described in earlier work on blockwise adaptivity [5, 26] and in [10.

We introduce the multiplicative and additive Schwarz overlapping domain decomposition methods in §2. We present the a posteriori error analysis in $\$ 3$. Examples are provided for multiplicative Schwarz in $\$ 4$ and for additive Schwarz in $\$ 5$. Details of the analysis appear in $\$ 6$. A discussion and future research directions appear in $\$ 7$.

\section{Overlapping Schwarz domain decomposition}

Assume that we have $p$ overlapping subdomains $\Omega_{1}, \cdots, \Omega_{p}$ on a domain $\Omega$, such that for any $\Omega_{i}$, there exists a $\Omega_{j}, i \neq j$, for which $\Omega_{i} \cap \Omega_{j} \neq \emptyset$ and $\cup_{i} \Omega_{i}=\Omega$. We use $L_{2}(\Omega)$ to denote the space of square integrable functions, $H^{1}(\Omega)$ for functions having a $L_{2}(\Omega)$ derivative and $H_{0}^{1}(\Omega)$ as the subspace of $H^{1}(\Omega)$ of functions satisfying homogeneous Dirichlet boundary conditions. We let $(\cdot, \cdot)$ and $(\cdot, \cdot)_{i j}$ represent the $L_{2}(\Omega)$ and $L_{2}\left(\Omega_{i} \cap \Omega_{j}\right)$ inner products respectively.

Consider the weak form of a second-order linear elliptic partial differential equation (PDE) problem: find $u \in H_{0}^{1}(\Omega)$ such that

$$
a(u, v)=l(v) \quad \forall v \in H_{0}^{1}(\Omega) .
$$

Here $a(\cdot, \cdot)$ is the standard bilinear form over $\Omega$ arising from integration by parts of the PDE operator and $l(\cdot)$ is the linear functional arising from the right-hand-side of the PDE. For example, given the Poisson equation $-\nabla^{2} u(x)=f(x)$ with homogeneous Dirichlet boundary conditions, we have $a(u, v)=\int_{\Omega} \nabla u \cdot \nabla v d x$ and $l(v)=(f, v)$. We denote by $a_{i}(\cdot, \cdot)$ the restriction of $a(\cdot, \cdot)$ to $\Omega_{i}$ and $a_{i j}(\cdot, \cdot)$ the restriction of $a(\cdot, \cdot)$ to $\Omega_{i} \cap \Omega_{j}$. Similarly, we let $l_{i}(\cdot)$ be the restriction of $l(\cdot)$ to $\Omega_{i}$.

We are interested in a QoI which is a linear functional of the solution,

$$
Q(u)=(\psi, u),
$$

where $\psi \in L_{2}(\Omega)$.

\subsection{Multiplicative Schwarz overlapping domain decomposition}

Defining $H_{D_{k}}^{1}\left(\Omega_{i}\right) \equiv\left\{v \in H^{1}\left(\Omega_{i}\right) \mid v=u^{\{k+(i-1) / p\}}\right.$ on $\left.\partial \Omega_{i}\right\}$, we present the multiplicative Schwarz method in Algorithm 1 . 


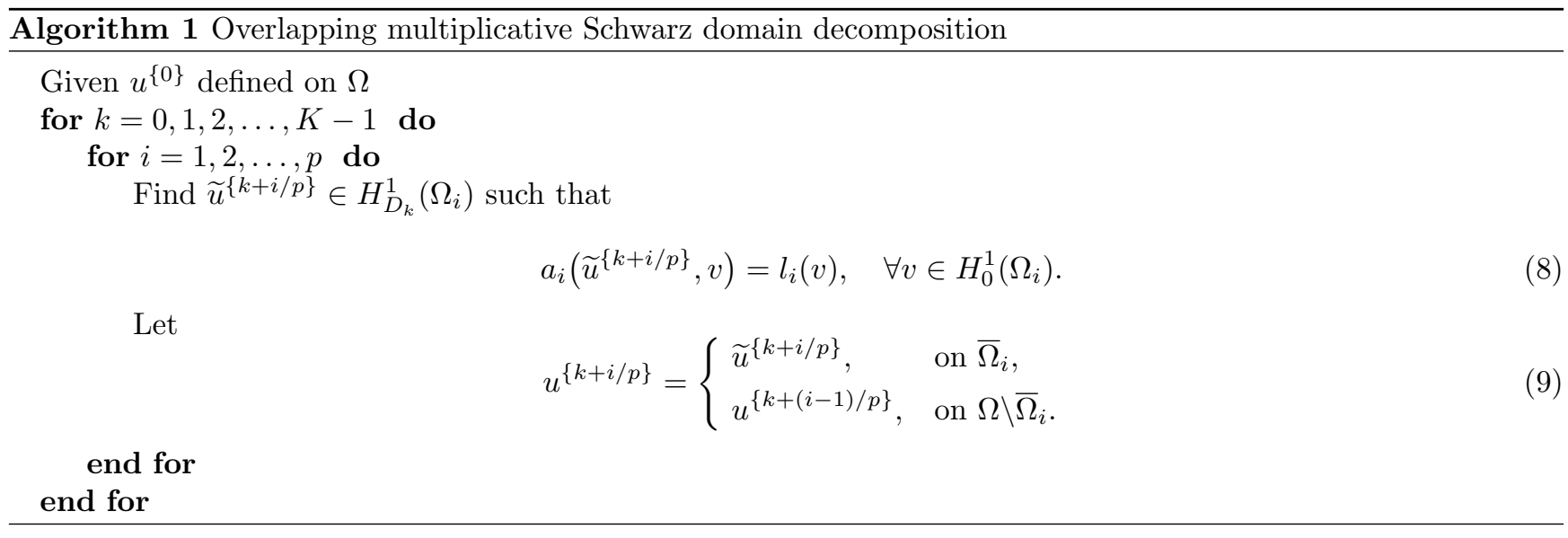

\subsection{Additive Schwarz overlapping domain decomposition}

The additive Schwarz solution method is given in Algorithm 2 with $H_{D_{k}}^{1}\left(\Omega_{i}\right) \equiv\left\{v \in H_{D}^{1}\left(\Omega_{i}\right) \mid v=u^{\{k\}}\right.$ on $\left.\partial \Omega_{i}\right\}$. The Richardson parameter $\tau$, is needed to ensure that the iteration converges [32].

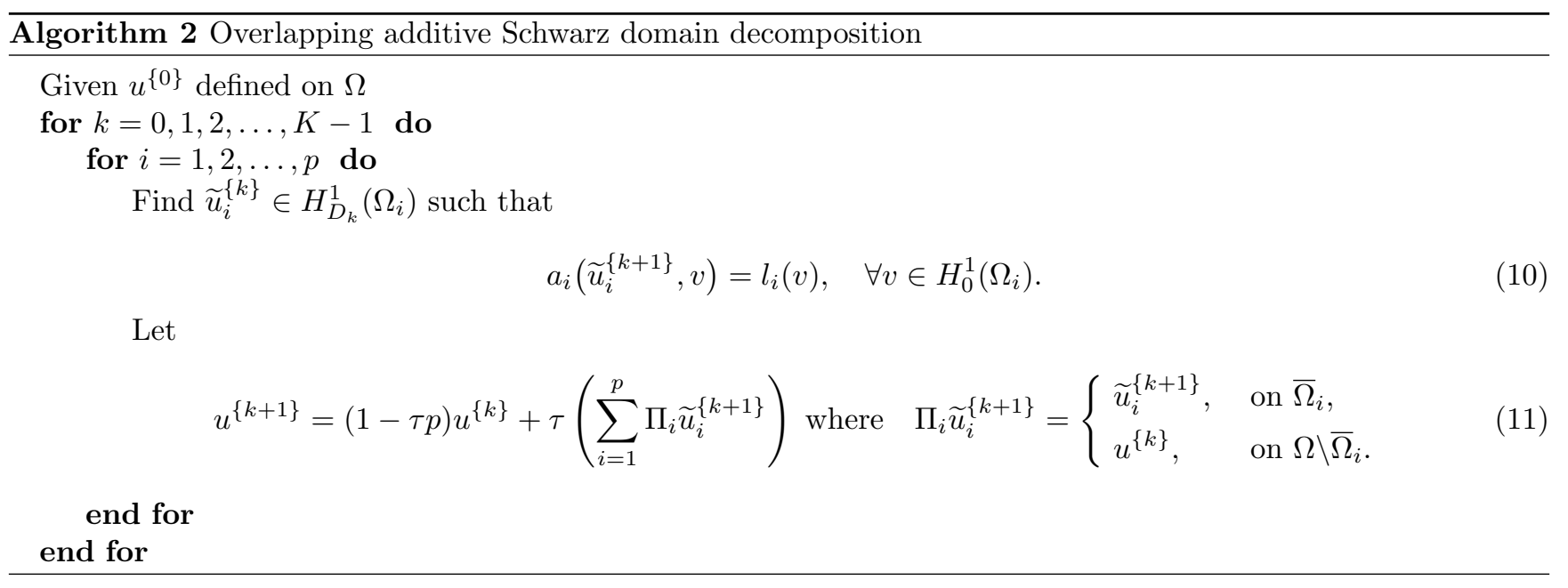

\subsection{Finite element discretizations}

We let $\mathcal{T}_{h}=\left\{T_{m}\right\}_{m=1}^{M}$ denote a quasi-regular triangulation of $\Omega$ in to non-overlapping elements $T_{m}$ such that no node of one element $T_{i}$ intersects an interior edge of another element $T_{j}$, and $\Omega=\cup_{m} T_{m}$. Moreover, the triangulation is consistent with the domain decomposition in the sense that if $T_{i} \cap \Omega_{j} \neq \emptyset$ then $T_{i} \subset \Omega_{j}$. The discretization of the overlapping domain decomposition approximation substitutes finite dimensional spaces $V_{i, h}^{k}$ for $H_{D_{k}}^{1}\left(\Omega_{i}\right)$ and $V_{i, h, 0}$ for $H_{0}^{1}\left(\Omega_{i}\right)$ in Algorithm 1, where $V_{i, h}^{k}$ and $V_{i, h, 0}$ refer to the standard finite element spaces consisting space of continuous piecewise polynomial functions on $\mathcal{T}_{h, i}=\left.\mathcal{T}_{h}\right|_{\Omega_{i}}$. Additionally, $V_{h} \subset H_{0}^{1}(\Omega)$ is the finite element space consisting of continuous piecewise polynomial functions with respect to $\mathcal{T}_{h}$.

We represent the global discrete solutions as $U^{\{k+i / p\}}$ (resp. $U_{i}^{\{k\}}$ ) belonging to the space $V_{h}$ and the local discrete solutions as $\widetilde{U}^{\{k+i / p\}}$ (resp. $\widetilde{U}_{i}^{\{k\}}$ ) belonging to the space $V_{i, h, 0}$ for the multiplicative (resp. additive) Schwarz methods. For simplicity we assume that $U^{\{0\}}=u^{\{0\}}$. For both algorithms, the global continuum, (resp. discrete), solution after $k$ iterations is represented as $u^{\{k\}}$, (resp. $\left.U^{\{k\}}\right)$.

\section{A posteriori analysis for the finite element approximation computed using Schwarz algorithms}

We derive a representation formula for the error in the QoI, $Q(u)-Q\left(U^{\{K\}}\right)=\left(\psi, u-U^{\{K\}}\right)$, that is computed from the discrete solution of the multiplicative or additive domain decomposition method after $K$ iterations. 


\subsection{The total error and its components}

We first consider the total numerical error, and then its decomposition into discretization and iteration error components.

\subsubsection{The total error}

We define the global adjoint

$$
a(v, \phi)=(\psi, v), \quad \forall v \in H_{0}^{1}(\Omega) .
$$

Theorem 1 (Total error representation). The error in the QoI for the discretized multiplicative or additive Schwarz algorithm after $K$ iterations is given by

$$
\left(u-U^{\{K\}}, \psi\right)=R\left(U^{\{K\}}, \phi\right),
$$

where $R\left(U^{\{K\}}, \phi\right)=l(\phi)-a\left(U^{\{K\}}, \phi\right)$ is the weak residual.

The proof of Theorem 1 is standard, see e.g., [19]. Unfortunately, it does not capture the structure of the differential operator corresponding to the Schwarz domain decomposition, which is reflected in the lack of Galerkin orthogonality in the expression. Performing Schwarz domain decomposition with a finite number of iterations defines a differential operator which is different than the differential operator associated with the original PDE (6). The numerical solution $U^{\{K\}}$ is a solution to the discretization of this modified operator. We carry out an analysis by decomposing the error into two contributions: iterative and discretization errors. For implementation purposes we note that the global adjoint $\phi$ is solved using a higher order finite element scheme. The global adjoint may be approximated by a Schwarz domain decomposition method provided sufficient iterations are performed, or the overlap is sufficiently large that the iteration error is negligible.

\subsubsection{Discretization and iteration errors}

We decompose the total error as

$$
u-U^{\{K\}}=\underbrace{u-u^{\{K\}}}_{\text {Iteration Error }}+\underbrace{u^{\{K\}}-U^{\{K\}}}_{\text {Discretization Error }}=e_{I}^{\{K\}}+e_{D}^{\{K\}},
$$

where $e_{I}^{\{k\}}=u-u^{\{k\}}, e_{D}^{\{k\}}=u^{\{k\}}-U^{\{k\}}$ and $e_{D}^{\{0\}}=0$. The iteration error quantifies the error due to the discrepancy between the PDE differential operator and the modified differential operator in the Schwarz algorithms arising from using a finite number $(K)$ iterations. The discretization error arises from the discretization of the modified differential operator.

Theorem 2 (Iteration error representation). We have

$$
\left(u-u^{\{K\}}, \psi\right)=R\left(U^{\{K\}}, \phi\right)-\left(\psi, u^{\{K\}}-U^{\{K\}}\right) .
$$

Proof. This follows by combining (13) and (14).

The analysis involves partitioning of the QoI data over subdomains by a partition of unity. Similar ideas were used in [23]. Let $\left\{\chi_{i}\right\}_{i=1}^{p}$ be a partition of unity such that

$$
\psi_{i}=\chi_{i} \psi,
$$

and $\psi_{i}=0$ on $\Omega \backslash \Omega_{i}$. The partition of unity localizes the QoI data to the subdomains. Let $d_{i}(x)$ denote the distance function

$$
d_{i}(x)=\left\{\begin{array}{r}
\operatorname{dist}\left(x, B^{(i)}\right), \text { if } x \in \bar{\Omega}_{i} \\
0, \text { if } x \notin \bar{\Omega}_{i},
\end{array}\right.
$$

where $B^{(i)} \equiv\left(\partial \Omega_{i} \cap \Omega\right)$. Then set

$$
\chi_{i}(x)=\frac{d_{i}(x)}{\sum_{j=1}^{p} d_{j}(x)} .
$$

With the partition of the QoI data, we have the following partition of the QoI. 
Lemma 1 (Partitioning the QoI data over subdomains). We have

$$
\left(e_{D}^{\{k\}}, \psi\right)=\sum_{i=1}^{p}\left(e_{D}^{\{k\}}, \psi_{i}\right)_{i i}
$$

Proof. This follows directly from the definition of the partition of unity in (16),

$$
\left(e_{D}^{\{k\}}, \psi\right)=\left(e_{D}^{\{k\}}, \sum_{i=1}^{p} \chi_{i} \psi\right)=\sum_{i=1}^{p}\left(e_{D}^{\{k\}}, \psi_{i}\right)_{i i} .
$$

\subsubsection{Weak Residuals}

We define the subdomain weak residual for a function $s$,

$$
R_{i}(s, v)=l_{i}(v)-a_{i}(s, v)
$$

for $i=1,2, \ldots, p$.

\subsection{A posteriori error analysis of discretization error for multiplicative Schwarz}

In this section, we derive a representation of the discretization error, $\left(\psi, u^{\{K\}}-U^{\{K\}}\right)$, for the multiplicative Schwarz method.

\subsubsection{Adjoint problems}

Define solutions $\phi^{[k+i / p]} \in H_{0}^{1}\left(\Omega_{i}\right)$ of the adjoint problems,

$$
\left\{\begin{array}{l}
a_{p}\left(v, \phi^{[Q+i / p]}\right)=\tau_{p}^{Q}(v), \quad \forall v \in H_{0}^{1}\left(\Omega_{p}\right), \\
a_{i}\left(v, \phi^{[Q+i / p]}\right)=\tau_{i}^{Q}(v)-\sum_{j=i+1}^{p} a_{i j}\left(v, \phi^{[Q+j / p]}\right), \quad 1 \leq i \leq p, \quad \forall v \in H_{0}^{1}\left(\Omega_{i}\right),
\end{array}\right.
$$

where

$$
\tau_{i}^{Q}(v)=\left\{\begin{array}{l}
\sum_{j=1}^{p}\left(v, \psi_{j}\right)_{i j}, \quad Q=K-1, \\
-\sum_{j=1}^{i-1} a_{i j}\left(v, \phi^{[Q+1+j / p]}\right), \quad 0 \leq Q<K-1 .
\end{array}\right.
$$

The right hand side of 21 captures not only the residuals corresponding to the localized QoI data (in the form of $\left.\left(v, \psi_{j}\right)_{i j}\right)$, but also the transfer error between subdomains as the iteration proceeds (in the form of $\left.-\sum_{j=1}^{i-1} a_{i j}\left(v, \phi^{[Q+1+j / p]}\right)-\sum_{j=i+1}^{p} a_{i j}\left(v, \phi^{[Q+j / p]}\right)\right)$. The adjoint problems 21] have the same sequential nature of subdomains solves as the multiplicative Schwarz Algorithm 1], but note that these are defined backwards from $K, K-1+(p-1) / p, K-1+(p-2) / p, \cdots, 1$.

\subsubsection{Discretization error}

Theorem 3 (Discretization error for multiplicative Schwarz). We have

$$
\left(\psi, u^{\{K\}}-U^{\{K\}}\right)=\sum_{k=0}^{K-1} \sum_{i=1}^{p} R_{i}\left(\widetilde{U}^{\{k+i / p\}}, \phi^{[k+i / p]}-\pi_{i} \phi^{[k+i / p]}\right),
$$

where $\pi_{i} v$ is the approximation of $v \in H_{0}^{1}\left(\Omega_{i}\right)$ in $V_{i, h, 0}$.

The proof of Theorem 3 is presented as a sequence of lemmas in 86.2 The term $\left(\phi^{[k+i / p]}-\pi_{i} \phi^{[k+i / p]}\right)$ arises from the use of Galerkin orthogonality, or the fact that the residual of the discrete solution is zero on the finite dimensional space $V_{i, h, 0}$. This reflects the fact that $\widetilde{U}^{\{k+i / p\}}$ is the discrete approximation to $u^{\{k+i / p\}}$, not to $u$. 


\subsection{A posteriori analysis of the discretization error for additive Schwarz}

In this section, we derive representation of the discretization error in the QoI obtained from the additive Schwarz method.

\subsubsection{Adjoint problems for discretization error}

Define $\phi_{i}^{[k]} \in H_{0}^{1}\left(\Omega_{i}\right)$ solutions to the adjoint problems,

$$
a_{i}\left(v, \phi_{i}^{[k]}\right)=\tau \sum_{j=1}^{p}\left\{\left(\psi_{j}, v\right)_{i j}-a_{i j}\left(v, \sum_{l=k+1}^{K} \phi_{j}^{[l]}\right)\right\}, \quad \forall v \in H_{0}^{1}\left(\Omega_{i}\right) .
$$

For a fixed $k$, the adjoint problems (24) are independent for each $i$, so $\phi_{i}^{[k]}$ may be computed backwards from $K, K-1, K-2, \cdots, 1$ in parallel analogous to the solution strategy in the additive Schwarz Algorithm 2 . We also note that for implementation purposes $\sum_{l=k+1}^{K} \phi_{j}^{[l]}$ involves a sum of the vectors, $\sum_{l=k+2}^{K} \phi_{j}^{[l]}$ (computed earlier) and $\phi_{j}^{[k+1]}$.

\subsubsection{Discretization error}

Theorem 4 (Discretization error for additive Schwarz). We have

$$
\left(\psi, e_{D}^{\{K\}}\right)=\left(\psi, u^{\{K\}}-U^{\{K\}}\right)=\sum_{k=1}^{K} \sum_{i=1}^{p} R_{i}\left(\widetilde{U}_{i}^{\{k\}}, \phi_{i}^{[k]}-\pi_{i} \phi_{i}^{[k]}\right) .
$$

The proof of Theorem 4 is presented as a sequence of lemmas in $\$ 6.3$.

\subsection{Solution algorithms}

The full algorithm for a posteriori error estimation for overlapping multiplicative/additive Schwarz domain decomposition is provided in Algorithm 3.

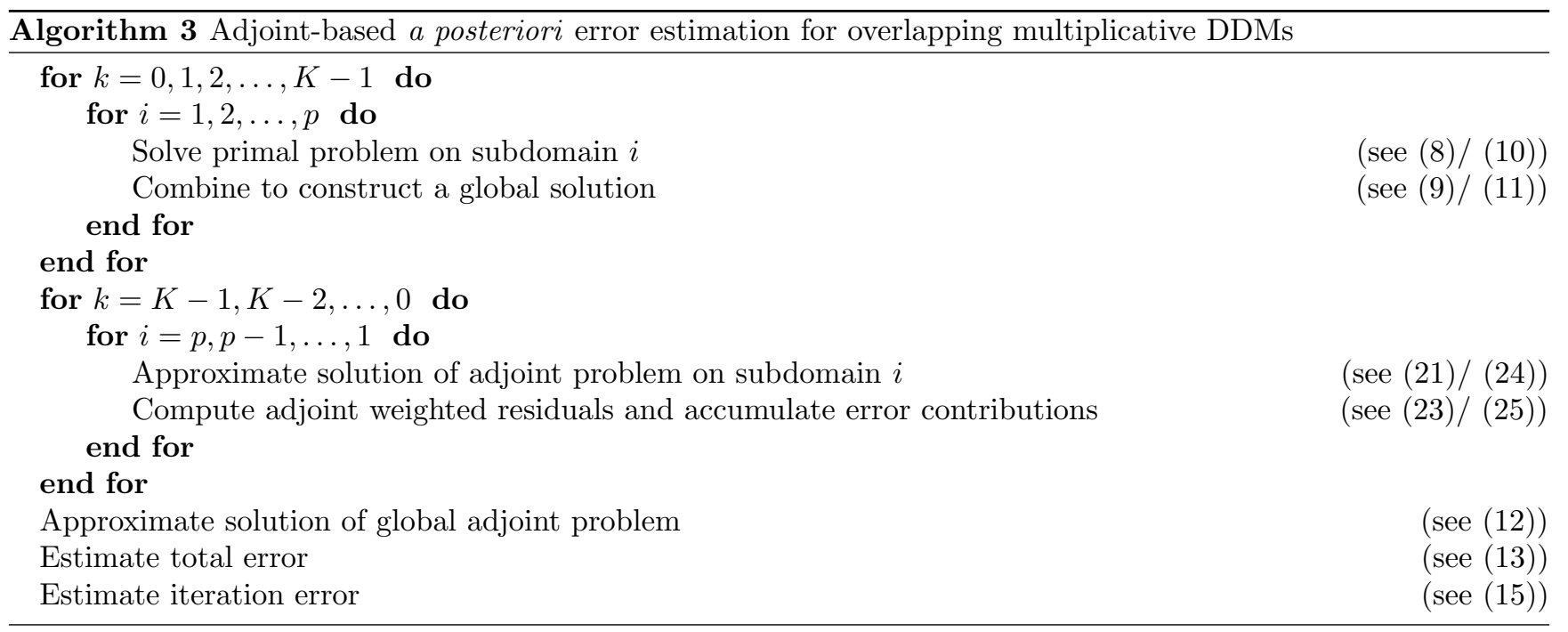

\section{Numerical examples for multiplicative Schwarz}

We provide examples for both multiplicative and additive Schwarz in order to demonstrate the accuracy of the a posteriori error estimate for a range of scenarios, stressing the importance of the ability to distinguish the contributions from discretization and iteration. The initial examples in 4.2 are chosen to illustrate certain expected behaviors. We expect the discretization error to decrease as the mesh is refined, and the iteration error to decrease as we increase the number of iterations or the degree of overlap. We expect the discretization error to be constant 
if the mesh is fixed when the number of subdomains is increased, but expect the iteration error to increase. In other words, the discretization error is determined by the mesh, but the iteration error is determined by the number (and disposition) of subdomains, the degree of overlap, and the number of iterations. In $\$ 4.3$, we construct a problem where the discretization and iteration errors have opposite signs, and show that iterating with a fixed mesh may result in the overall error initially decreasing as the iteration error decreases, achieving a minimum when the discretization and iteration errors cancel each other, and then increasing (and stabilizing) as the discretization error comes to dominate the total error. For the convection dominated problem in $\$ 4.4$, we show how the configuration of the subdomains affects the iteration error, but not the discretization error. Finally in $\$ 4.5$ we provide two examples of a two stage strategy in which an accurate error estimate for an initial coarse solution guides the construction of a more accurate "production" calculation. We choose to locally adapt the finite element mesh when the discretization error in a particular subdomain is dominant, and to increase the degree of subdomain overlap when iteration is the leading source of error. "Adaptivity" in the context of iterative methods, requires strategies for addressing both discretization and iteration errors.

\subsection{Error estimates and effectivity ratios}

We compute approximate adjoint solutions $\Phi^{[k+i / p]} \approx \phi^{[k+i / p]}$, and $\Phi \approx \phi$ and then compute (23) and (13). The resulting error estimates are

$$
\eta_{D}^{K} \equiv \sum_{k=0}^{K-1} \sum_{i=1}^{p} R_{i}\left(\widetilde{U}^{\{k+i / p\}}, \Phi^{[k+i / p]}-\pi_{i} \Phi^{[k+i / p]}\right),
$$

and

$$
\eta^{K} \equiv R\left(U^{\{K\}}, \Phi\right) .
$$

One way to measure the performance of an error estimates is the "effectivity ratios",

$$
\gamma=\frac{\eta^{K}}{\left(u-U^{\{K\}}, \psi\right)},
$$

and

$$
\gamma_{D}=\frac{\eta_{D}^{K}}{\left(u^{\{K\}}-U^{\{K\}}, \psi\right)} .
$$

An effectivity ratio close to one indicates that the error estimate is accurate. We also recall that $e_{I}$ denotes the iteration error.

\subsection{Poisson's equation}

Consider Poisson's equation

$$
\begin{aligned}
-\nabla^{2} u=f, & & \text { in } \Omega, \\
u=0, & & \text { on } \partial \Omega,
\end{aligned}
$$

in a square domain $\Omega=[0,1] \times[0,1]$, where $f(x, y)=8 \pi^{2} \sin (2 \pi x) \sin (2 \pi y)$. The QoI in (7) is specified by

$$
\psi=\mathbb{1}_{[.6, .8] \times[.6, .8]} .
$$

where $\mathbb{1}_{\omega}$ is the characteristic function on a domain $\omega$. In the computations below, unless otherwise specified, the mesh is uniform and contains $2 \times N_{x} \times N_{y}$ triangular elements. The overlap between subdomains is indicated by $\beta$.

\subsection{1 $2 \times 1$ subdomains}

Two overlapping subdomains $\Omega_{1}=[0, .6] \times[0,1]$ and $\Omega_{2}=[.4,1] \times[0,1]$ are illustrated in Figure 1a corresponding to an overlap parameter $\beta=0.1$. The solid black lines in this figure and in subsequent figures, indicate the center line between overlapping subdomains.

Estimates of the discretization, iteration and total errors, and the corresponding effectivity ratios as we vary the overlap $\beta$, number of Schwarz iterations $K$ and number of elements are shown in Tables 1. In all cases the effectivity ratios are close to 1.0. The table highlights the sensitivity of the estimates to the different contributions of the error. The "base" computation with $N_{x}=N_{y}=20, \beta=0.1$ and $K=2$ is repeated for ease of comparison. 


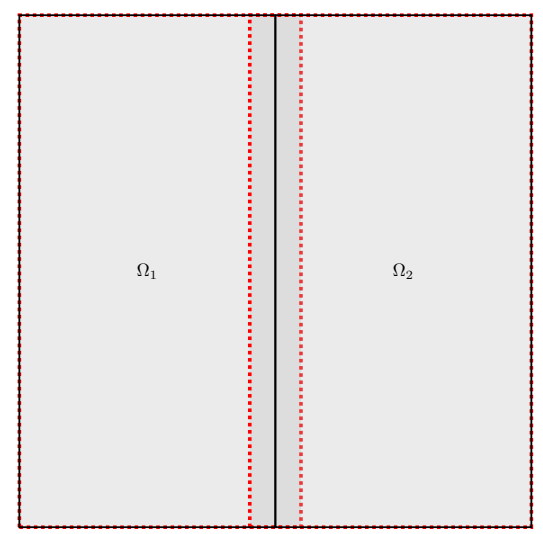

(a)

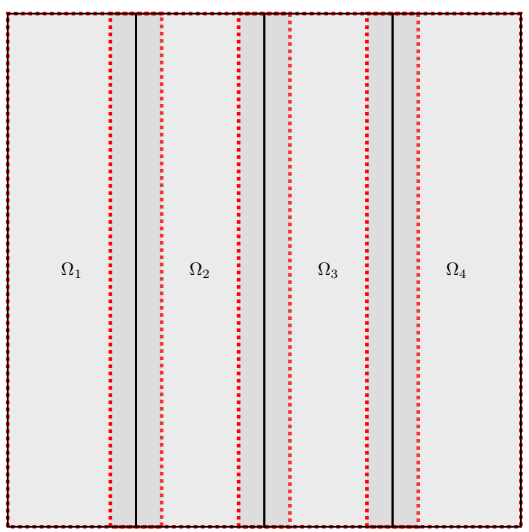

(b)

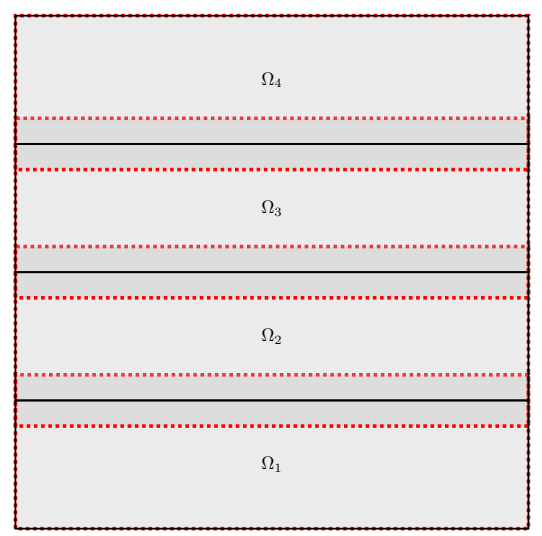

(c)

Figure 1: Overlapping subdomains with $\beta=0.1$. (a) Two $(2 \times 1)$ subdomains. (b) Four $(4 \times 1)$ subdomains. (c) Four $(1 \times 4)$ subdomains.

Increasing the overlap decreases the iteration error $e_{I}^{\{K\}}$ but does not have a significant effect on the discretization error $e_{D}^{\{K\}}$. The iteration error decreases with increasing number of Schwarz iterations, but the discretization error is largely unaffected when the mesh is fixed. The discretization error decreases when the mesh is refined with a fixed number of iterations, but the iteration error remains essentially constant.

\begin{tabular}{||c|c|c|c|c|c|c|c|c||}
\hline$N_{x}$ & $N_{y}$ & $\beta$ & $K$ & Est. Err. & $\gamma$ & $e_{D}^{\{K\}}$ & $\gamma_{D}$ & $e_{I}^{\{K\}}$ \\
\hline 20 & 20 & 0.1 & 2 & $1.02 \mathrm{e}-03$ & $9.98 \mathrm{E}-01$ & $6.56 \mathrm{e}-04$ & $9.98 \mathrm{E}-01$ & $3.60 \mathrm{e}-04$ \\
20 & 20 & 0.2 & 2 & $7.03 \mathrm{e}-04$ & $9.96 \mathrm{E}-01$ & $6.28 \mathrm{e}-04$ & $9.97 \mathrm{E}-01$ & $7.50 \mathrm{e}-05$ \\
\hline 20 & 20 & 0.1 & 2 & $1.02 \mathrm{e}-03$ & $9.98 \mathrm{E}-01$ & $6.56 \mathrm{e}-04$ & $9.98 \mathrm{E}-01$ & $3.60 \mathrm{e}-04$ \\
20 & 20 & 0.1 & 4 & $6.55 \mathrm{e}-04$ & $9.96 \mathrm{E}-01$ & $6.26 \mathrm{e}-04$ & $9.97 \mathrm{E}-01$ & $2.89 \mathrm{e}-05$ \\
\hline 20 & 20 & 0.1 & 2 & $1.02 \mathrm{e}-03$ & $9.98 \mathrm{E}-01$ & $6.56 \mathrm{e}-04$ & $9.98 \mathrm{E}-01$ & $3.60 \mathrm{e}-04$ \\
40 & 40 & 0.1 & 2 & $5.25 \mathrm{e}-04$ & $1.00 \mathrm{E}+00$ & $1.66 \mathrm{e}-04$ & $9.99 \mathrm{E}-01$ & $3.60 \mathrm{e}-04$ \\
\hline
\end{tabular}

Table 1: Multiplicative Schwarz for Poisson's equation: $2 \times 1$ subdomains.

\subsection{2 $4 \times 1$ subdomains}

The computational domains for $\beta=0.1$ are shown in Figure $1 \mathrm{~b}$, It is well known that as the number of subdomains increases, the convergence of Schwarz methods decreases, and this is evident by comparing Tables 2 and 1 . While the discretization errors of the four subdomain and two subdomain cases are comparable in magnitude, the iteration error $e_{I}^{\{K\}}$ is an order of magnitude larger for four subdomains compared to two. The contributions of the separate components of the total error vary with the overlap, number of iterations and number of elements in a qualitatively similar way to the results in $\S 4.2 .1$.

\begin{tabular}{||c|c|c|c|c|c|c|c|c||}
\hline$N_{x}$ & $N_{y}$ & $\beta$ & $K$ & Est. Err. & $\gamma$ & $e_{D}^{\{K\}}$ & $\gamma_{D}$ & $e_{I}^{\{K\}}$ \\
\hline 20 & 20 & 0.1 & 2 & $4.57 \mathrm{e}-03$ & $9.99 \mathrm{e}-01$ & $6.92 \mathrm{e}-04$ & $9.97 \mathrm{e}-01$ & $3.88 \mathrm{e}-03$ \\
20 & 20 & 0.2 & 2 & $1.34 \mathrm{e}-03$ & $9.98 \mathrm{e}-01$ & $6.48 \mathrm{e}-04$ & $9.98 \mathrm{e}-01$ & $6.87 \mathrm{e}-04$ \\
\hline 20 & 20 & 0.1 & 2 & $4.57 \mathrm{e}-03$ & $9.99 \mathrm{e}-01$ & $6.92 \mathrm{e}-04$ & $9.97 \mathrm{e}-01$ & $3.88 \mathrm{e}-03$ \\
20 & 20 & 0.1 & 4 & $1.04 \mathrm{e}-03$ & $9.98 \mathrm{e}-01$ & $6.48 \mathrm{e}-04$ & $9.98 \mathrm{e}-01$ & $3.94 \mathrm{e}-04$ \\
\hline 20 & 20 & 0.1 & 2 & $4.57 \mathrm{e}-03$ & $9.99 \mathrm{e}-01$ & $6.92 \mathrm{e}-04$ & $9.97 \mathrm{e}-01$ & $3.88 \mathrm{e}-03$ \\
40 & 40 & 0.1 & 2 & $4.05 \mathrm{e}-03$ & $1.00 \mathrm{e}+00$ & $1.75 \mathrm{e}-04$ & $9.99 \mathrm{e}-01$ & $3.88 \mathrm{e}-03$ \\
\hline
\end{tabular}

Table 2: Multiplicative Schwarz for Poisson's equation: $4 \times 1$ subdomains. 


\subsection{3 $4 \times 4$ subdomains}

The computational domains for $\beta=0.1$ and sixteen equally-sized subdomains are configured in a $4 \times 4$ grid, see Figure 2a. The error estimates are quite accurate. The results, shown in Table 3 are qualitatively similar to those in Tables 1 and 2 The iteration error is larger than in the $4 \times 1$ case, while the discretization errors are essentially the same in both, which is to be expected since the finite element meshes are the same.

\begin{tabular}{||c|c|c|c|c|c|c|c|c||}
\hline$N_{x}$ & $N_{y}$ & $\beta$ & $K$ & Est. Err. & $\gamma$ & $e_{D}^{\{K\}}$ & $\gamma_{D}$ & $e_{I}^{\{K\}}$ \\
\hline 20 & 20 & 0.1 & 2 & $9.22 \mathrm{e}-03$ & $1.00 \mathrm{e}+00$ & $1.02 \mathrm{e}-03$ & $1.00 \mathrm{e}+00$ & $8.20 \mathrm{e}-03$ \\
20 & 20 & 0.2 & 2 & $2.80 \mathrm{e}-03$ & $9.99 \mathrm{e}-01$ & $7.31 \mathrm{e}-04$ & $9.98 \mathrm{e}-01$ & $2.07 \mathrm{e}-03$ \\
\hline 20 & 20 & 0.1 & 2 & $9.22 \mathrm{e}-03$ & $1.00 \mathrm{e}+00$ & $1.02 \mathrm{e}-03$ & $1.00 \mathrm{e}+00$ & $8.20 \mathrm{e}-03$ \\
20 & 20 & 0.1 & 4 & $2.72 \mathrm{e}-03$ & $9.99 \mathrm{e}-01$ & $8.27 \mathrm{e}-04$ & $9.99 \mathrm{e}-01$ & $1.90 \mathrm{e}-03$ \\
\hline 20 & 20 & 0.1 & 2 & $9.22 \mathrm{e}-03$ & $1.00 \mathrm{e}+00$ & $1.02 \mathrm{e}-03$ & $1.00 \mathrm{e}+00$ & $8.20 \mathrm{e}-03$ \\
40 & 40 & 0.1 & 2 & $8.45 \mathrm{e}-03$ & $1.00 \mathrm{e}+00$ & $2.55 \mathrm{e}-04$ & $1.00 \mathrm{e}+00$ & $8.20 \mathrm{e}-03$ \\
\hline
\end{tabular}

Table 3: Multiplicative Schwarz for Poisson's equation: $4 \times 4$ subdomains.

\subsection{Cancellation of error}

To illustrate the potential for cancellation between discretization and iteration errors, the quantity of interest is chosen to be

$$
\psi=\mathbb{1}_{[.4, .8] \times[.4, .8]} .
$$

for two subdomains and an overlap $\beta=0.05$. Computational results for an increasing number of Schwarz iterations are shown in Table 4. The magnitude of the total error initially decreases as the iteration proceeds, reaching a minimum after six iterations, but then starts to increase. This behavior is explained by observing that the discretization and iteration errors have opposite signs. The discretization error is essentially fixed as the iteration proceeds and has a value of $-1.6 \times 10^{-4}$. The initial iteration error is of order $4.0 \times 10^{-3}$ and dominates the total error. As expected, the iteration error decreases monotonically as $K$ increases, but is always positive. After six iterations the discretization and iteration errors have approximately equal magnitudes but opposite signs, and cancel to produce a total error of $3.0 \times 10^{-5}$. For greater than six iterations, the iteration error continues to decrease and now the discretization error dominates the total error. The total error increases to $-1.5 \times 10^{-4}$ after 10 iterations and gradually approaches the (fixed) discretization error as the number of iterations increases further.

\begin{tabular}{||c|c|c|c|c|c||}
\hline$K$ & Est. Err. & $\gamma$ & $e_{D}^{\{K\}}$ & $\gamma_{D}$ & $e_{I}^{\{K\}}$ \\
\hline 1 & $3.98 \mathrm{e}-03$ & $1.00 \mathrm{e}+00$ & $-1.50 \mathrm{e}-05$ & $9.98 \mathrm{e}-01$ & $4.00 \mathrm{e}-03$ \\
2 & $2.07 \mathrm{e}-03$ & $1.00 \mathrm{e}+00$ & $-5.95 \mathrm{e}-05$ & $9.99 \mathrm{e}-01$ & $2.13 \mathrm{e}-03$ \\
3 & $1.04 \mathrm{e}-03$ & $1.00 \mathrm{e}+00$ & $-9.11 \mathrm{e}-05$ & $1.00 \mathrm{e}+00$ & $1.13 \mathrm{e}-03$ \\
4 & $4.89 \mathrm{e}-04$ & $1.00 \mathrm{e}+00$ & $-1.14 \mathrm{e}-04$ & $1.00 \mathrm{e}+00$ & $6.03 \mathrm{e}-04$ \\
5 & $1.91 \mathrm{e}-04$ & $1.00 \mathrm{e}+00$ & $-1.30 \mathrm{e}-04$ & $1.00 \mathrm{e}+00$ & $3.21 \mathrm{e}-04$ \\
6 & $2.97 \mathrm{e}-05$ & $1.01 \mathrm{e}+00$ & $-1.41 \mathrm{e}-04$ & $1.00 \mathrm{e}+00$ & $1.71 \mathrm{e}-04$ \\
7 & $-5.83 \mathrm{e}-05$ & $9.96 \mathrm{e}-01$ & $-1.49 \mathrm{e}-04$ & $1.00 \mathrm{e}+00$ & $9.09 \mathrm{e}-05$ \\
8 & $-1.07 \mathrm{e}-04$ & $9.98 \mathrm{e}-01$ & $-1.55 \mathrm{e}-04$ & $1.00 \mathrm{e}+00$ & $4.84 \mathrm{e}-05$ \\
9 & $-1.33 \mathrm{e}-04$ & $9.98 \mathrm{e}-01$ & $-1.59 \mathrm{e}-04$ & $1.00 \mathrm{e}+00$ & $2.58 \mathrm{e}-05$ \\
10 & $-1.48 \mathrm{e}-04$ & $9.98 \mathrm{e}-01$ & $-1.62 \mathrm{e}-04$ & $1.00 \mathrm{e}+00$ & $1.37 \mathrm{e}-05$ \\
\hline
\end{tabular}

Table 4: Multiplicative Schwarz for Poisson's equation: $2 \times 1$ subdomains, $N_{x}=N_{y}=40, \beta=0.05$.

\subsection{A convection-diffusion problem}

Consider the convection-diffusion equation,

$$
\begin{aligned}
-\nabla^{2} u+\mathbf{b} \cdot \nabla u & =f, & & \text { in } \Omega, \\
u & =0, & & \text { on } \partial \Omega,
\end{aligned}
$$

where $\Omega=[0,1] \times[0,1], f(x, y)=1$, and $\mathbf{b}=[-60,0]$. The effect of the convection is that a perturbation to data on the right affects the solution to the left. For this example, we choose the quantity of interest

$$
\psi=\mathbb{1}_{[.05, .2] \times[.05, .2]},
$$


concentrated near the bottom left hand corner. The adjoint problems are solved using continuous piecewise cubic polynomials to ensure accurate solutions in the presence of the strong convective vector field. We experiment with two configurations with the subdomains aligned with different coordinate axes, and either parallel with or perpendicular to the direction of convection.

\subsection{1 $4 \times 1$ configuration}

This subdomain configuration is the same as in Figure $1 \mathrm{~b}$. The total, discretization and iteration errors are provided in Table 5. Note the significant iteration error in this configuration for $K=2$, which dominates the total error. The large iteration error for $K=2$ is to be expected given direction of information travel from right to left. The iteration error decreases dramatically for $K=4$ and $K=6$, and discretization error becomes the dominant error.

\subsection{2 $1 \times 4$ configuration}

This subdomain configuration is shown in Figure 1c. The subdomains are aligned with the direction of the convective vector field. The iteration error after two iterations and the total error are more than an order of magnitude less than in the $4 \times 1$ case. In this scenario, one subdomain contains most of the "domain of influence" for the QoI [23. and hence results in low iteration error, even for $K=2$. There is again cancellation between the discretization and iteration errors for $K=2$ so that the total error increases for $K=4$ and $K=6$ with the total error dominated by the discretization error.

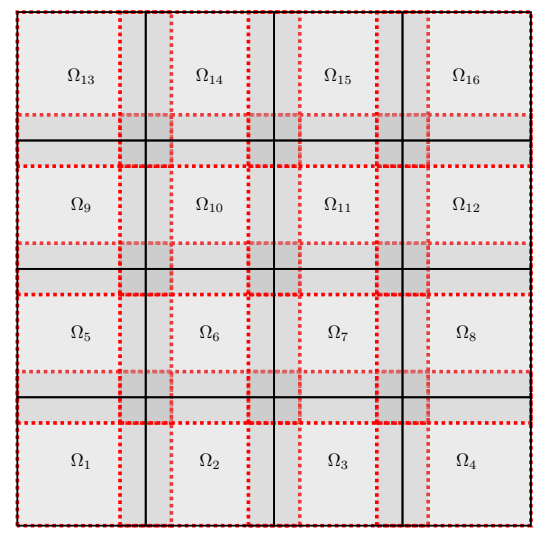

(a)

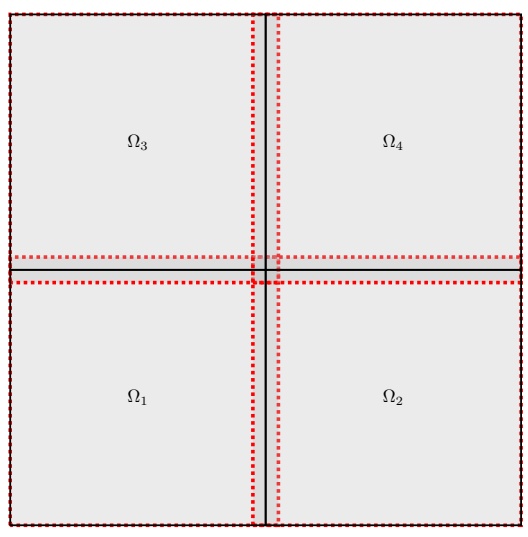

(b)

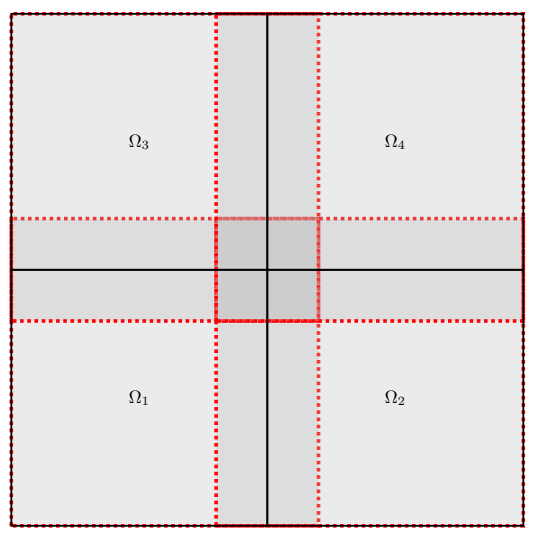

(c)

Figure 2: (a) Sixteen $(4 \times 4)$ overlapping subdomains with $\beta=0.1$. (b) Four $(2 \times 2)$ overlapping subdomains with $\beta=0.05$. (c) Four $(2 \times 2)$ overlapping subdomains with $\beta=0.2$.

\begin{tabular}{||c|c|c|c|c|c||}
\hline$K$ & Est. Err. & $\gamma$ & $e_{D}^{\{K\}}$ & $\gamma_{D}$ & $e_{I}^{\{K\}}$ \\
\hline \multicolumn{7}{|c||}{$4 \times 1$ configuration } \\
\hline 2 & $9.76 \mathrm{e}-03$ & $1.00 \mathrm{e}+00$ & $-1.54 \mathrm{e}-04$ & $9.87 \mathrm{e}-01$ & $9.92 \mathrm{e}-03$ \\
4 & $-1.15 \mathrm{e}-04$ & $9.81 \mathrm{e}-01$ & $-1.42 \mathrm{e}-04$ & $9.77 \mathrm{e}-01$ & $2.67 \mathrm{e}-05$ \\
6 & $-3.54 \mathrm{e}-04$ & $9.94 \mathrm{e}-01$ & $-3.54 \mathrm{e}-04$ & $9.94 \mathrm{e}-01$ & $4.36 \mathrm{e}-10$ \\
\hline \multicolumn{7}{|c|}{$1 \times 4$ configuration } \\
\hline 2 & $8.42 \mathrm{e}-05$ & $1.03 \mathrm{e}+00$ & $-3.73 \mathrm{e}-04$ & $9.94 \mathrm{e}-01$ & $4.57 \mathrm{e}-04$ \\
4 & $-3.54 \mathrm{e}-04$ & $9.94 \mathrm{e}-01$ & $-3.55 \mathrm{e}-04$ & $9.94 \mathrm{e}-01$ & $3.53 \mathrm{e}-07$ \\
6 & $-3.54 \mathrm{e}-04$ & $9.94 \mathrm{e}-01$ & $-3.54 \mathrm{e}-04$ & $9.94 \mathrm{e}-01$ & $3.70 \mathrm{e}-11$ \\
\hline
\end{tabular}

Table 5: Multiplicative Schwarz for convection-diffusion: $N_{x}=N_{y}=20, \beta=0.1$.

\subsection{Two stage solution strategy for Poisson's equation}

Adjoint-based a posteriori error estimates can provide useful information for designing efficient two stage strategies for computing approximate solutions. First, a preliminary, inexpensive computation is performed on a coarse discretization. The a posteriori error estimate for the "stage 1" solution is computed and the different error 
contributions determined. A more expensive "stage 2" approximation is computed using numerical parameters chosen to balance the sources of error. We provide two examples of this strategy below. The stage 1 computation for both experiments is run on a $2 \times 2$ subdomain configuration as shown in Figure $2 \mathrm{c}$.

\subsubsection{Dominant discretization error}

Consider the QoI given by (31). The results on the initial $2 \times 2$ subdomain configuration with $N_{x}=N_{y}=10, \beta=0.2$ and $K=6$ are provided in Table 6. The mesh for this computation is shown in Figure 3a. The main source of the error is the discretization error $e_{D}^{\{K\}}$. In order to reduce the discretization error, we need to reduce the discretization error contribution arising from each subdomain. We define the contribution to the discretization error from subdomain $i$ as

$$
S_{i}^{K}=\sum_{k=0}^{K-1} R_{i}\left(\widetilde{U}^{\{k+i / p\}}, \phi^{[k+i / p]}-\pi_{i} \phi^{[k+i / p]}\right), \quad i=1, \ldots, p,
$$

so that the discretization error, 23 may be written as

$$
\left(\psi, u^{\{K\}}-U^{\{K\}}\right)=\sum_{i=1}^{p} S_{i}^{K} .
$$

The values of $S_{i}^{K}$ for the stage 1 calculation are also shown in Table 6 . Subdomain 4 contributes the most towards the discretization error, and hence it is the prime candidate for refinement. After refining all the elements in subdomain 4, the refined mesh is shown in Figure 3b. The discretization errors in each subdomain $S_{i}^{K}$ and the total error after the refinement are shown in Table 6. The discretization error is significantly lower and hence the total error is also significantly lower. The values of $S_{i}^{K}$ also indicate that now each subdomain contributes roughly the same magnitude towards the discretization error.

We note that we can take advantage of cancellation of the discretization errors. Applying the standard approximation theory for degree one Lagrange finite elements, we expect the discretization error component $S_{4}^{K}$ to decrease by a factor of four if we refine the mesh corresponding to subdomain 4 uniformly. The conjectured value for $S_{4}^{K}$ is therefore approximately $9 \times 10^{-4}$. The discretization errors from subdomains 2 and 3 (represented by $S_{2}^{K}$ and $S_{3}^{K}$ ) have negative signs and are not expected to change as significantly when subdomain 4 is refined. As shown in Table 6, after refinement of subdomain 4, there is significant cancellation of error between subdomains 2 and 3 and subdomain 4 , and the total error is $3.44 \times 10^{-4}$. Uniformly refining the entire initial mesh results in a refined mesh with 441 vertices (shown in Figure 3c) and the solution after $K=6$ iterations has a total error of $6.24 \times 10^{-4}$, which is approximately the expected four-fold reduction in error. The mesh refined using adjoint based error information in Figure $3 \mathrm{~b}$ has almost half the number of degree of freedoms of the uniformly refined mesh in Figure 3c, but none-the-less has half the total error $\left(3.44 \times 10^{-4}\right.$ vs. $\left.6.24 \times 10^{-4}\right)$. Recognizing and taking advantage of cancellation of error can produce otherwise startling efficiencies. Similar refinement strategies, where specific "components" are refined to exploit cancellation of error, are also employed in [9, 10, Such component-wise refinement strategies allow for estimation of the decrease of error in a more reliable manner than classical adaptive refinement strategies in which disparate elements are marked for refinement.

\begin{tabular}{||c|c|c|c|c|c|c||}
\hline Stage & Num. vertices & Est. Err. & $\gamma$ & $e_{D}^{\{K\}}$ & $\gamma_{D}$ & $e_{I}^{\{K\}}$ \\
\hline 1 & 121 & $2.36 \mathrm{e}-03$ & $9.83 \mathrm{e}-01$ & $2.36 \mathrm{e}-03$ & $9.89 \mathrm{e}-01$ & $6.98 \mathrm{e}-06$ \\
2 & 253 & $3.44 \mathrm{e}-04$ & $1.00 \mathrm{e}+00$ & $3.37 \mathrm{e}-04$ & $1.05 \mathrm{e}+00$ & $6.97 \mathrm{e}-06$ \\
\hline Stage & Num. vertices & $i$ & 1 & 2 & 3 & 4 \\
\hline 1 & 121 & $S_{i}^{K}$ & $3.07 \mathrm{e}-04$ & $-7.94 \mathrm{e}-04$ & $-7.82 \mathrm{e}-04$ & $3.62 \mathrm{e}-03$ \\
2 & 253 & $S_{i}^{K}$ & $1.82 \mathrm{e}-04$ & $-3.87 \mathrm{e}-04$ & $-3.85 \mathrm{e}-04$ & $9.27 \mathrm{e}-04$ \\
\hline
\end{tabular}

Table 6: Two stage solution strategy using multiplicative Schwarz to solve Poisson's equation: $\beta=0.2, K=6$.

\subsubsection{Dominant iteration error}

For the same choice of QoI, we perform a stage 1 computation with $2 \times 2$ subdomains and $N_{x}=N_{y}=40, \beta=0.05$ and $K=2$. This configuration is shown in Figure $2 \mathrm{~b}$. The contributions to the total error are shown in Table 7. The dominant source of the error is the iteration error $e_{I}^{\{K\}}$. There are two ways to reduce it, either by performing a great number of iterations or increasing $\beta$. We choose the latter option and set $\beta=0.2$, see Figure $2 \mathrm{c}$. The 


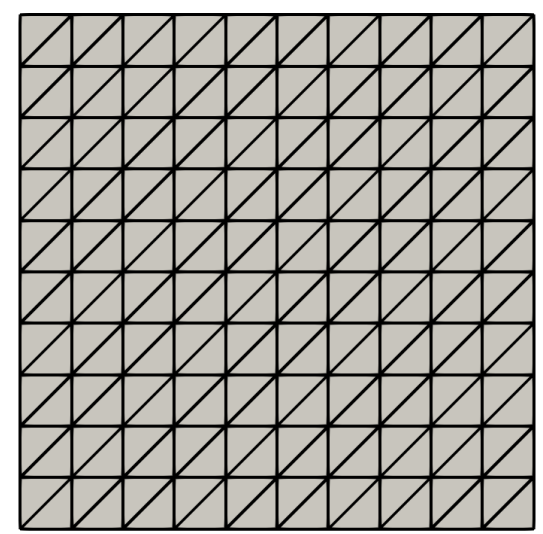

(a)

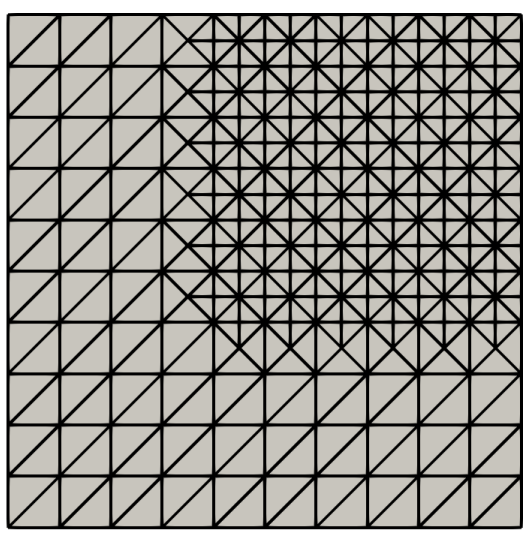

(b)

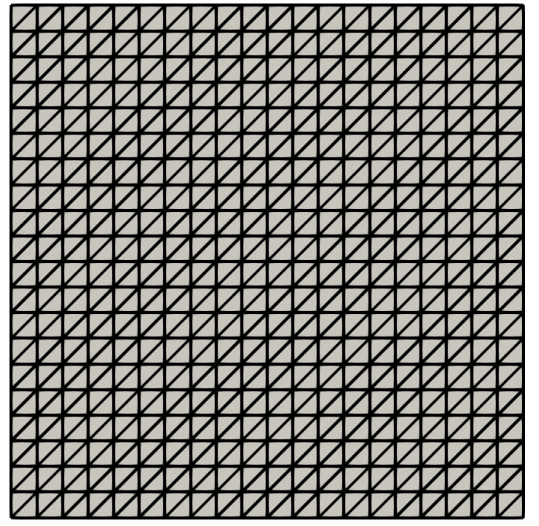

(c)

Figure 3: (a) Initial uniform mesh. (b) Mesh refinement in $\Omega_{4}$ guided by adjoint based error estimates. (c) Uniformly refined mesh.

results are shown in Table 7, where now the iteration error and discretization are balanced and the overall error has decreased.

\begin{tabular}{||c|c|c|c|c|c|c|c|c|c||}
\hline Stage & $N_{x}$ & $N_{y}$ & $\beta$ & $K$ & Est. Err. & $\gamma$ & $e_{D}^{\{K\}}$ & $\gamma_{D}$ & $e_{I}^{\{K\}}$ \\
\hline 1 & 40 & 40 & 0.05 & 2 & $1.23 \mathrm{e}-03$ & $1.00 \mathrm{e}+00$ & $1.79 \mathrm{e}-04$ & $9.99 \mathrm{e}-01$ & $1.05 \mathrm{e}-03$ \\
2 & 40 & 40 & 0.2 & 2 & $5.04 \mathrm{e}-04$ & $1.00 \mathrm{e}+00$ & $1.62 \mathrm{e}-04$ & $9.99 \mathrm{e}-01$ & $3.42 \mathrm{e}-04$ \\
\hline
\end{tabular}

Table 7: Two stage solution strategy using multiplicative Schwarz to solve Poisson's equation. Stage 1: $\beta=0.05$, stage $2: \beta=0.2$.

\section{$5 \quad$ Numerical examples for additive Schwarz}

We repeat analogous numerical examples in $\$ 4$ for additive Schwarz. Effectivity ratios for the discretization error and the total error are defined analogously to the case of the multiplicative Schwarz case by replacing $\Phi^{[k+i / p]}$ in the above expressions by $\Phi_{i}^{[k]}$ in the expressions in $\$ 4.1$, where $\Phi_{i}^{[k]}$ is the numerical approximation to $\phi_{i}^{[k]}$. A relaxation parameter $\tau=0.4$ was used in all examples. The error estimates are again highly accurate with effectivity ratios close to 1 .

\subsection{Estimates for Poisson's equation}

\subsection{1 $2 \times 1$ subdomains}

We solve the same problem described in 4.2 .1 by equations (30) and (31) using additive Schwarz. The results are shown in Table 8. In comparison to the results in 4.2 .1 , we observe that the additive Schwarz method has much higher iteration error than multiplicative Schwarz method. The discretization error is of course approximately the same.

\begin{tabular}{||c|c|c|c|c|c|c|c|c||}
\hline$N_{x}$ & $N_{y}$ & $\beta$ & $K$ & Est. Err. & $\gamma$ & $e_{D}^{\{K\}}$ & $\gamma_{D}$ & $e_{I}^{\{K\}}$ \\
\hline 20 & 20 & 0.1 & 2 & $1.09 \mathrm{e}-02$ & $1.00 \mathrm{e}+00$ & $4.52 \mathrm{e}-04$ & $9.98 \mathrm{e}-01$ & $1.05 \mathrm{e}-02$ \\
20 & 20 & 0.2 & 2 & $1.04 \mathrm{e}-02$ & $1.00 \mathrm{e}+00$ & $4.34 \mathrm{e}-04$ & $9.98 \mathrm{e}-01$ & $9.96 \mathrm{e}-03$ \\
\hline 20 & 20 & 0.1 & 2 & $1.09 \mathrm{e}-02$ & $1.00 \mathrm{e}+00$ & $4.52 \mathrm{e}-04$ & $9.98 \mathrm{e}-01$ & $1.05 \mathrm{e}-02$ \\
20 & 20 & 0.1 & 4 & $4.23 \mathrm{e}-03$ & $9.99 \mathrm{e}-01$ & $6.02 \mathrm{e}-04$ & $9.98 \mathrm{e}-01$ & $3.62 \mathrm{e}-03$ \\
\hline 20 & 20 & 0.1 & 2 & $1.09 \mathrm{e}-02$ & $1.00 \mathrm{e}+00$ & $4.52 \mathrm{e}-04$ & $9.98 \mathrm{e}-01$ & $1.05 \mathrm{e}-02$ \\
40 & 40 & 0.1 & 2 & $1.06 \mathrm{e}-02$ & $1.00 \mathrm{e}+00$ & $1.14 \mathrm{e}-04$ & $9.99 \mathrm{e}-01$ & $1.05 \mathrm{e}-02$ \\
\hline
\end{tabular}

Table 8: Additive Schwarz for Poisson's equation: $2 \times 1$ subdomains. 


\subsection{2 $4 \times 1$ subdomains}

The results solving the same problem using twice the number of subdomains are shown in Table 9, The iteration error is considerably larger than for multiplicative Schwarz and the convergence rate with increasing numbers of iterations appears to be much slower. The discretization error is again approximately the same.

\begin{tabular}{||c|c|c|c|c|c|c|c|c||}
\hline$N_{x}$ & $N_{y}$ & $\beta$ & $K$ & Est. Err. & $\gamma$ & $e_{D}^{\{K\}}$ & $\gamma_{D}$ & $e_{I}^{\{K\}}$ \\
\hline 20 & 20 & 0.1 & 2 & $1.89 \mathrm{e}-02$ & $1.00 \mathrm{e}+00$ & $5.42 \mathrm{e}-04$ & $9.96 \mathrm{e}-01$ & $1.84 \mathrm{e}-02$ \\
20 & 20 & 0.2 & 2 & $1.19 \mathrm{e}-02$ & $1.00 \mathrm{e}+00$ & $6.04 \mathrm{e}-04$ & $9.97 \mathrm{e}-01$ & $1.13 \mathrm{e}-02$ \\
\hline 20 & 20 & 0.1 & 2 & $1.89 \mathrm{e}-02$ & $1.00 \mathrm{e}+00$ & $5.42 \mathrm{e}-04$ & $9.96 \mathrm{e}-01$ & $1.84 \mathrm{e}-02$ \\
20 & 20 & 0.1 & 4 & $1.21 \mathrm{e}-02$ & $1.00 \mathrm{e}+00$ & $6.51 \mathrm{e}-04$ & $9.97 \mathrm{e}-01$ & $1.14 \mathrm{e}-02$ \\
\hline 20 & 20 & 0.1 & 2 & $1.89 \mathrm{e}-02$ & $1.00 \mathrm{e}+00$ & $5.42 \mathrm{e}-04$ & $9.96 \mathrm{e}-01$ & $1.84 \mathrm{e}-02$ \\
40 & 40 & 0.1 & 2 & $1.85 \mathrm{e}-02$ & $1.00 \mathrm{e}+00$ & $1.38 \mathrm{e}-04$ & $9.99 \mathrm{e}-01$ & $1.84 \mathrm{e}-02$ \\
\hline
\end{tabular}

Table 9: Additive Schwarz for Poisson's equation: $4 \times 1$ subdomains.

\subsection{3 $4 \times 4$ subdomains}

Repeating the problem in 4.2 .3 and using additive Schwarz produces the results provided in Table 10

\begin{tabular}{||c|c|c|c|c|c|c|c|c||}
\hline$N_{x}$ & $N_{y}$ & $\beta$ & $K$ & Est. Err. & $\gamma$ & $e_{D}^{\{K\}}$ & $\gamma_{D}$ & $e_{I}^{\{K\}}$ \\
\hline 20 & 20 & 0.1 & 2 & $2.18 \mathrm{e}-02$ & $1.00 \mathrm{e}+00$ & $6.83 \mathrm{e}-04$ & $1.00 \mathrm{e}+00$ & $2.12 \mathrm{e}-02$ \\
20 & 20 & 0.2 & 2 & $1.25 \mathrm{e}-02$ & $1.00 \mathrm{e}+00$ & $6.14 \mathrm{e}-04$ & $1.00 \mathrm{e}+00$ & $1.18 \mathrm{e}-02$ \\
\hline 20 & 20 & 0.1 & 2 & $2.18 \mathrm{e}-02$ & $1.00 \mathrm{e}+00$ & $6.83 \mathrm{e}-04$ & $1.00 \mathrm{e}+00$ & $2.12 \mathrm{e}-02$ \\
20 & 20 & 0.1 & 4 & $1.58 \mathrm{e}-02$ & $1.00 \mathrm{e}+00$ & $9.42 \mathrm{e}-04$ & $9.86 \mathrm{e}-01$ & $1.48 \mathrm{e}-02$ \\
\hline 20 & 20 & 0.1 & 2 & $2.18 \mathrm{e}-02$ & $1.00 \mathrm{e}+00$ & $6.83 \mathrm{e}-04$ & $1.00 \mathrm{e}+00$ & $2.12 \mathrm{e}-02$ \\
40 & 40 & 0.1 & 2 & $2.13 \mathrm{e}-02$ & $1.00 \mathrm{e}+00$ & $1.70 \mathrm{e}-04$ & $1.00 \mathrm{e}+00$ & $2.12 \mathrm{e}-02$ \\
\hline
\end{tabular}

Table 10: Additive Schwarz for Poisson's equation: $4 \times 4$ subdomains.

Once again the iteration error is significantly greater than in the multiplicative case and appears to improve more slowly with increasing overlap or number of iterations.

\subsection{A convection-diffusion problem}

The problem formulation is defined in $\$ 4.4$ by equations 33 and (34). We provide results for two different configurations of the subdomains in Table 11 below.

\begin{tabular}{||c|c|c|c|c|c||}
\hline$K$ & Est. Err. & $\gamma$ & $e_{D}^{\{K\}}$ & $\gamma_{D}$ & $e_{I}^{\{K\}}$ \\
\hline \multicolumn{7}{|c||}{$4 \times 1$ configuration } \\
\hline 2 & $1.78 \mathrm{e}-02$ & $1.00 \mathrm{e}+00$ & $-1.04 \mathrm{e}-04$ & $9.92 \mathrm{e}-01$ & $1.79 \mathrm{e}-02$ \\
4 & $1.28 \mathrm{e}-02$ & $1.00 \mathrm{e}+00$ & $-9.38 \mathrm{e}-05$ & $9.81 \mathrm{e}-01$ & $1.29 \mathrm{e}-02$ \\
6 & $8.40 \mathrm{e}-03$ & $1.00 \mathrm{e}+00$ & $-5.56 \mathrm{e}-05$ & $9.54 \mathrm{e}-01$ & $8.46 \mathrm{e}-03$ \\
\hline \multicolumn{7}{||c}{$1 \times 4$ configuration } \\
\hline 2 & $1.08 \mathrm{e}-02$ & $1.00 \mathrm{e}+00$ & $-1.32 \mathrm{e}-04$ & $9.91 \mathrm{e}-01$ & $1.10 \mathrm{e}-02$ \\
4 & $5.11 \mathrm{e}-03$ & $1.00 \mathrm{e}+00$ & $-2.37 \mathrm{e}-04$ & $9.93 \mathrm{e}-01$ & $5.35 \mathrm{e}-03$ \\
6 & $2.32 \mathrm{e}-03$ & $1.00 \mathrm{e}+00$ & $-3.01 \mathrm{e}-04$ & $9.94 \mathrm{e}-01$ & $2.62 \mathrm{e}-03$ \\
\hline
\end{tabular}

Table 11: Additive Schwarz for convection-diffusion: $N_{x}=N_{y}=20, \beta=0.1$.

The differences between these two configurations are not as dramatic as in the case of multiplicative Schwarz. Furthermore, both $4 \times 1$ and $1 \times 4$ configurations had essentially converged after 6 iterations of multiplicative Schwarz. This is far from true for additive Schwarz. 


\subsection{Two stage solution strategy for Poisson's equation}

\subsubsection{Dominant discretization error}

We repeat the problem in 84.5 .1 and the results are shown in Table 12 . We observe the expected reduction in discretization error in subdomain 4 , but the reduction in total error is not as dramatic as in the multiplicative Schwarz case. There is less cancellation between discretization errors of opposite sign following local mesh refinement, and the iteration error is much larger for additive Schwarz. However, after mesh refinement in subdomain 4 and six iterations, the iteration error makes the largest contribution to the total error.

\begin{tabular}{||c|c|c|c|c|c|c||}
\hline Stage & Num. vertices & Est. Err. & $\gamma$ & $e_{D}^{\{K\}}$ & $\gamma_{D}$ & $e_{I}^{\{K\}}$ \\
\hline 1 & 121 & $3.24 \mathrm{e}-03$ & $9.88 \mathrm{e}-01$ & $2.48 \mathrm{e}-03$ & $9.90 \mathrm{e}-01$ & $7.61 \mathrm{e}-04$ \\
2 & 253 & $1.24 \mathrm{e}-03$ & $1.00 \mathrm{e}+00$ & $4.79 \mathrm{e}-04$ & $1.04 \mathrm{e}+00$ & $7.60 \mathrm{e}-04$ \\
\hline Stage & Num. vertices & $i$ & 1 & 2 & 3 & 4 \\
\hline 1 & 121 & $S_{i}^{K}$ & $6.82 \mathrm{e}-05$ & $-3.76 \mathrm{e}-04$ & $-3.76 \mathrm{e}-04$ & $3.16 \mathrm{e}-03$ \\
2 & 253 & $S_{i}^{K}$ & $4.13 \mathrm{e}-05$ & $-1.78 \mathrm{e}-04$ & $-1.78 \mathrm{e}-04$ & $7.93 \mathrm{e}-04$ \\
\hline
\end{tabular}

Table 12: Two stage solution strategy using additive Schwarz to solve Poisson's equation: $\beta=0.2, K=6$.

\subsubsection{Dominant iteration error}

The results upon repeating the problem in 4.5 .2 are shown in Table 13 Increasing the overlap reduces the iteration error, but not as effectively as for multiplicative Schwarz, and after only two iterations the iteration error remains significantly larger than the discretization error.

\begin{tabular}{||c|c|c|c|c|c|c|c|c||}
\hline$N_{x}$ & $N_{y}$ & $\beta$ & $K$ & Est. Err. & $\gamma$ & $e_{D}^{\{K\}}$ & $\gamma_{D}$ & $e_{I}^{\{K\}}$ \\
\hline 40 & 40 & 0.05 & 2 & $1.05 \mathrm{e}-02$ & $1.00 \mathrm{e}+00$ & $1.19 \mathrm{e}-04$ & $1.00 \mathrm{e}+00$ & $1.04 \mathrm{e}-02$ \\
40 & 40 & 0.2 & 2 & $8.27 \mathrm{e}-03$ & $1.00 \mathrm{e}+00$ & $1.15 \mathrm{e}-04$ & $1.00 \mathrm{e}+00$ & $8.15 \mathrm{e}-03$ \\
\hline
\end{tabular}

Table 13: Two stage solution strategy using additive Schwarz to solve Poisson's equation. Stage $1: \beta=0.05$, stage $2: \beta=0.2$.

\section{Details of analysis: algorithm reformulation, technical lemmas and proofs}

\subsection{Analogy with algebraic Gauss-Seidel iteration}

It is helpful to consider an algebraic analog of multiplicative Schwarz overlapping domain decomposition in order to provide insight in to the unusual forms of equations (21) and (22) and Theorem 3 . Consider solving the algebraic linear system $M x=b$, where $M$ is a $p \times p$ matrix, using $K$ Gauss-Seidel iterations. (Here we choose $p=4$ and $K=5$.)

We decompose the matrix $M$ as a sum of strictly lower triangular, diagonal and a strictly upper triangular matrices as $M=L+D+U$ and solve

$$
(L+D) x^{\{k+1\}}=b-U x^{\{k\}}, k=0,1, \ldots
$$

Let $A=(L+D)$ and $B=U$, each of which are $p \times p$ matrices. The complete Gauss-Seidel iteration can be written as the following block lower triangular system

$$
C_{\mathrm{gs}} \boldsymbol{x}=\boldsymbol{b}
$$

where

$$
C_{\mathrm{gS}}=\left[\begin{array}{ccccc}
A & 0 & 0 & 0 & 0 \\
B & A & 0 & 0 & 0 \\
0 & B & A & 0 & 0 \\
0 & 0 & B & A & 0 \\
0 & 0 & 0 & B & A
\end{array}\right]
$$


$C_{\mathrm{gs}}$ has a block bandwidth of two. Here,

$$
\boldsymbol{x}^{\top}=\left(x^{\{1\}^{\top}}, x^{\{2\}^{\top}}, x^{\{3\}^{\top}}, x^{\{4\}^{\top}}, x^{\{5\}^{\top}}\right) \quad \text { and } \quad \boldsymbol{b}^{\top}=\left(b^{\top}, b^{\top}, b^{\top}, b^{\top}, b^{\top}\right) .
$$

The corresponding adjoint problem is $C_{\mathrm{gS}}^{\top} \boldsymbol{\phi}=\boldsymbol{\psi}$ where $\boldsymbol{\psi}^{\top}=\left(0,0,0,0, \psi^{\top}\right)$, and

$$
C_{\mathrm{gS}}^{\top}=\left[\begin{array}{ccccc}
A^{\top} & B^{\top} & 0 & 0 & 0 \\
0 & A^{\top} & B^{\top} & 0 & 0 \\
0 & 0 & A^{\top} & B^{\top} & 0 \\
0 & 0 & 0 & A^{\top} & B^{\top} \\
0 & 0 & 0 & 0 & A^{\top}
\end{array}\right] .
$$

Note that the adjoint is non-zero only for the final solutions $x^{\{K\}}=x^{\{5\}}$. Let

$$
\phi^{\top}=\left(\phi_{1}, \phi_{2}, \phi_{3}, \phi_{4}, \phi_{5}\right)^{\top} \text {. }
$$

The adjoint problems are

$$
\begin{aligned}
& A^{\top} \boldsymbol{\phi}_{5}=\psi, \\
& A^{\top} \boldsymbol{\phi}_{4}=-B^{\top} \boldsymbol{\phi}_{5}, A^{\top} \boldsymbol{\phi}_{3}=-B^{\top} \boldsymbol{\phi}_{4}, A^{\top} \boldsymbol{\phi}_{2}=-B^{\top} \boldsymbol{\phi}_{3}, A^{\top} \boldsymbol{\phi}_{1}=-B^{\top} \boldsymbol{\phi}_{2},
\end{aligned}
$$

which can be solved sequentially by backward substitution. Recall that $A$ is lower triangular and $B$ is strictly upper triangular, hence

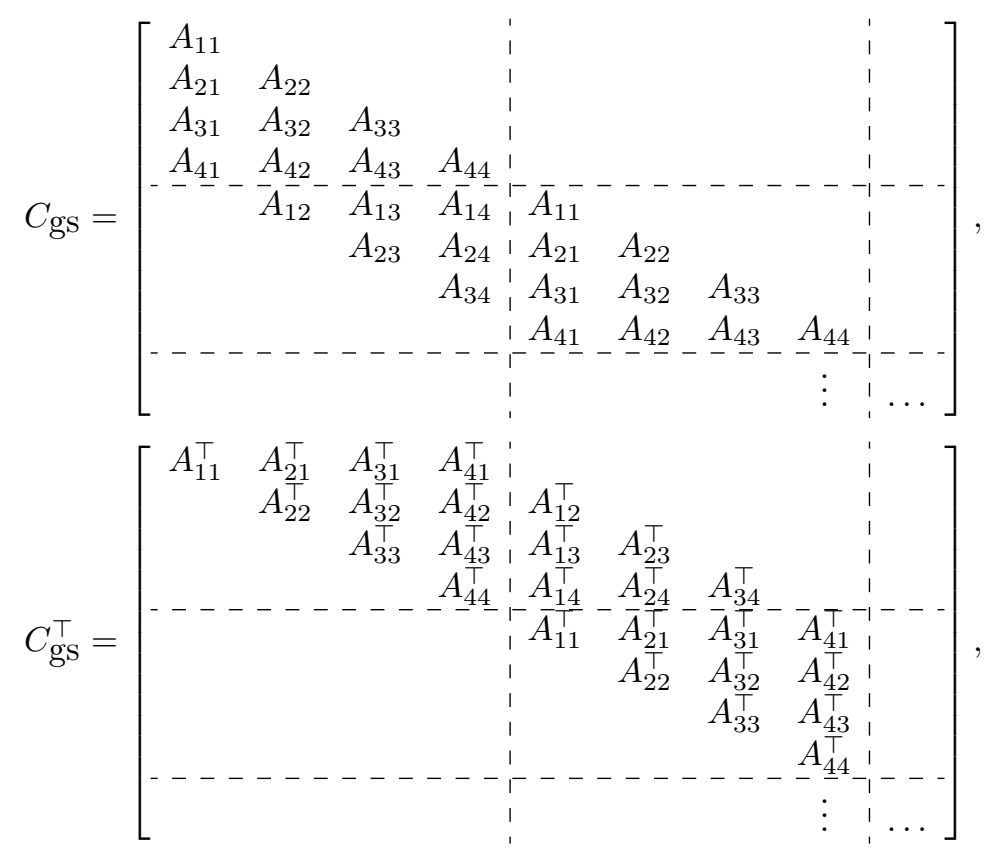

and the adjoint equations within each block can also be solved via backward substitution. The adjoint equations are therefore

$$
\begin{aligned}
& A_{i i}^{\top} \phi_{i}^{[K]}=\psi_{i}-\sum_{j>i} A_{j i}^{\top} \phi_{j}^{[K]}, \quad i=1, \ldots p, \\
& A_{i i}^{\top} \phi_{i}^{[k]}=-\sum_{j<i} A_{j i}^{\top} \phi_{j}^{[k+1]}-\sum_{j>i} A_{j i}^{\top} \phi_{j}^{[k]}, \quad i=1, \ldots p, \quad k=K-1, \ldots, 1 .
\end{aligned}
$$

The form of the adjoint problems in (37) and (38) mimic those in equations 221) and 22). The sum on the RHS of (37) represents the additional adjoint problems that must be solved to estimate effect of errors made while solving forward problems during the final $(K t h)$ iteration. We call these within iteration transfer errors. The first sum on the RHS of (38) represents the additional adjoint problems that must be solved to estimate the effect of errors made while solving forward problems during the previous iteration. We call these between iteration transfer errors. The second sum on the RHS of (38) again represents additional adjoint problems to estimate within iteration transfer 
errors. These two distinct types of transfer error were earlier identified in the context of operator decomposition approaches to coupled semilinear elliptic systems in [6]. Since $C_{\mathrm{gS}} \boldsymbol{x}=\boldsymbol{b}$ is just a $K p \times K p$ linear system,

$$
(e, \psi)=(\mathcal{R}, \phi)
$$

where

$$
\mathcal{R}=\boldsymbol{b}-C_{\mathrm{gS}} \hat{\boldsymbol{x}}
$$

for approximate solution $\hat{\boldsymbol{x}}$. The error in the quantity of interest can be expressed as an inner products of two vectors of length $K p$, and $(39)$ mimics the result in equation (23).

\subsection{Details of analysis of multiplicative Schwarz algorithm}

\subsubsection{Reformulation of the algorithm}

Algorithm 1 is not amenable to adjoint based analysis since the affine solution space $H_{D_{k}}^{1}\left(\Omega_{i}\right)$ changes at every iteration. We reformulate the algorithm by using a standard lifting technique to account for this in Algorithm 4. We set

$$
\widetilde{u}^{\{k+i / p\}}=w^{\{k+i / p\}}+u^{\{k+(i-1) / p\}} \quad \text { on } \Omega_{i},
$$

where $w^{\{k+i / p\}} \in H_{0}^{1}\left(\Omega_{i}\right)$.

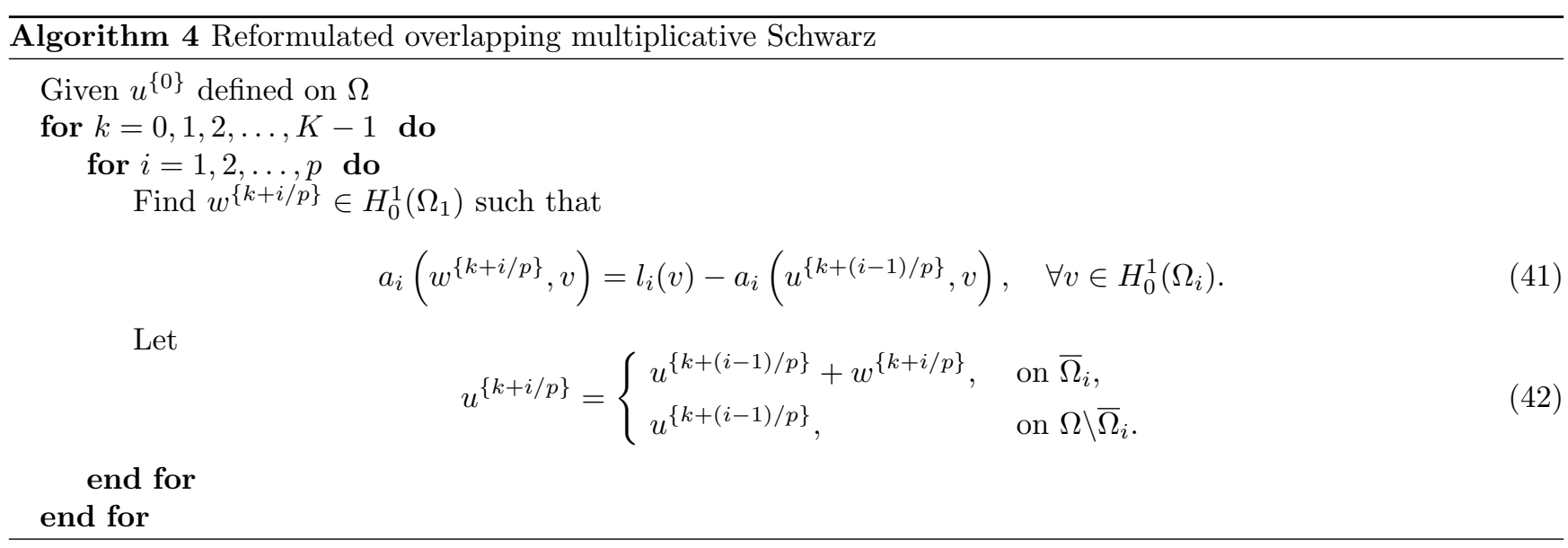

There is an equivalent reformulation of the discrete Algorithm 4 and we denote the unknown solutions as $W^{\{k+i / p\}}$ belonging to the spaces $V_{i, h, 0} \subset H_{0}^{1}\left(\Omega_{i}\right)$. The solutions $W^{\{k+i / p\}}$ are defined formally for the analysis but are not computed in practice.

To distinguish between different solutions (true, analytical, discrete) we use the notation in Table 14 .

\begin{tabular}{||c|c|c|l||}
\hline Notation & Formula & Space & Meaning \\
\hline$u$ & & $H_{0}^{1}(\Omega)$ & True solution \\
$u^{\{k\}}$ & & $H_{0}^{1}\left(\Omega_{i}\right)$ & Global analytic solution at iteration $k$ \\
$U^{\{k\}}$ & & $V_{h}$ & Global discrete solution at iteration $k$ \\
\hline$\widetilde{u}^{\{k+i / p\}}$ & & $H_{D_{k}}^{1}\left(\Omega_{i}\right)$ & Analytic solutions on $\Omega_{i}$ at iteration $k$ \\
$\widetilde{U}^{\{k+i / p\}}$ & & $V_{i, h}^{k}$ & Discrete solutions on $\Omega_{i}$ at iteration $k$ \\
\hline$w^{\{k+i / p\}}$ & & $H_{0}^{1}\left(\Omega_{i}\right)$ & Analytic solns on $\Omega_{i}$ with homogen. bcs at iteration $k$ \\
$W^{\{k+i / p\}}$ & & $V_{i, h, 0}$ & Discrete solns on $\Omega_{i}$ with homogen. bcs at iteration $k$ \\
\hline$e^{\{k\}}$ & $u-U^{\{k\}}$ & $H_{0}^{1}(\Omega)$ & Total error \\
$e_{I}^{\{k\}}$ & $u-u^{\{k\}}$ & $H_{0}^{1}(\Omega)$ & Global iteration error at iteration $k$ \\
$e_{D}^{\{k\}}$ & $u^{\{k\}}-U^{\{k\}}$ & $H_{0}^{1}(\Omega)$ & Global discretization error at iteration $k$ \\
\hline$e_{W}^{\{k+i / p\}}$ & $w^{\{k+i / p\}}-W^{\{k+i / p\}}$ & $H_{0}^{1}\left(\Omega_{i}\right)$ & Discretization error on $\Omega_{i}$ with homogen. bcs at it. $k$ \\
\hline
\end{tabular}

Table 14: Multiplicative Schwarz: notation for different solutions and their spaces. 


\subsubsection{Technical lemmas}

Let $e_{W}^{\{k\}}=w^{\{k\}}-W^{\{k\}}$. By 40 we have

$$
e_{W}^{\{k+i / p\}}=e_{D}^{\{k+i / p\}}-e_{D}^{\{k+(i-1) / p\}} \text { on } \Omega_{i} .
$$

Note that $e_{W}^{\{k+i / p\}}=0$ on $\partial \Omega_{i}$. We set $e_{W}^{\{k+i / p\}}=0$ on $\Omega \backslash \Omega_{i}$.

Lemma 2 (Error in QoI in terms of discretization errors with homogeneous bcs). The discretization error in the QoI is

$$
\left(e_{D}^{\{K\}}, \psi\right)=\sum_{k=0}^{K-1} \sum_{i=1}^{p} \sum_{j=1}^{p}\left(e_{W}^{\{k+i / p\}}, \psi_{j}\right)_{i j} .
$$

Proof. From equation (43) and the fact that $\psi_{j}=0$ on $\Omega \backslash \Omega_{j}$ for fixed $j$ we have

$$
\begin{aligned}
\left(e_{D}^{\{K\}}, \psi_{j}\right) & =\left(e_{D}^{\{K-1+p / p\}}, \psi_{j}\right) \\
& =\left(e_{W}^{\{K-i+p / p\}}, \psi_{j}\right)_{p j}+\left(e_{D}^{\{K-1+(p-1) / p\}}, \psi_{j}\right) \\
& =\left(e_{W}^{\{K-i+p / p\}}, \psi_{j}\right)_{p j}+\left(e_{W}^{\{K-i+(p-1) / p\}}, \psi_{j}\right)_{(p-1) j}+\left(e_{D}^{\{K-1+(p-2) / p\}}, \psi_{j}\right)
\end{aligned}
$$

Continuing,

$$
\left(e_{D}^{\{K\}}, \psi_{j}\right)=\left(e_{D}^{\{K-1\}}, \psi_{j}\right)+\sum_{i=1}^{p}\left(e_{W}^{\{K-1+i / p\}}, \psi_{j}\right)_{i j} .
$$

This is a recursive relation for $e_{D}^{\{K\}}$. Expanding $\left(e_{D}^{\{K-1\}}, \psi_{j}\right)$ as above leads to

$$
\left(e_{D}^{\{K\}}, \psi_{j}\right)=\sum_{k=0}^{K-1} \sum_{i=1}^{p}\left(e_{W}^{\{k+i / p\}}, \psi_{j}\right)_{i j} .
$$

Summing over $j=1, \ldots, p$,

$$
\sum_{j=1}^{p}\left(e_{D}^{\{K\}}, \psi_{j}\right)=\sum_{k=0}^{K-1} \sum_{i=1}^{p} \sum_{j=1}^{p}\left(e_{W}^{\{k+i / p\}}, \psi_{j}\right)_{i j}
$$

Lemma 3 (Bilinear form with discretization errors with homogeneous bcs). For any $v \in H_{D_{k}}^{1}\left(\Omega_{i}\right)$ we have

$$
a_{i}\left(e_{W}^{\{i / p\}}, v\right)=a_{i}\left(e_{D}^{\{i / p\}}, v\right)-\sum_{r=1}^{i-1} a_{i r}\left(e_{W}^{\{r / p\}}, v\right)
$$

and for $k \geq 1$,

$$
a_{i}\left(e_{W}^{\{k+i / p\}}, v\right)=a_{i}\left(e_{D}^{\{k+i / p\}}, v\right)-a_{i}\left(e_{D}^{\{k-1+i / p\}}, v\right)-\sum_{r=1}^{i-1} a_{i r}\left(e_{W}^{\{k+r / p\}}, v\right)-\sum_{r=i+1}^{p} a_{i r}\left(e_{W}^{\{k-1+r / p\}}, v\right) .
$$

Proof. By [43] we have for $m<i$,

$$
\begin{aligned}
a_{i}\left(e_{D}^{\{m / p\}}, v\right) & =a_{i}\left(e_{D}^{\{(m-1) / p\}}, v\right)+a_{i, m}\left(e_{W}^{\{m / p\}}, v\right), \\
& =a_{i}\left(e_{D}^{\{(m-2) / p\}}, v\right)+a_{i, m-1}\left(e_{W}^{\{(m-1) / p\}}, v\right)+a_{i, m}\left(e_{W}^{\{m / p\}}, v\right) .
\end{aligned}
$$

where we use $e_{W}^{\{r / p\}}=0$ on $\Omega \backslash \Omega_{r}$. Continuing in this manner yields

$$
a_{i}\left(e_{D}^{\{m / p\}}, v\right)=a_{i}\left(e_{D}^{\{0\}}, v\right)+\sum_{r=1}^{m} a_{i r}\left(e_{W}^{\{r / p\}}, v\right)=\sum_{r=1}^{m} a_{i r}\left(e_{W}^{\{r / p\}}, v\right)
$$


since $e_{D}^{\{0\}}=0$. Again by 43 ,

$$
a_{i}\left(e_{W}^{\{i / p\}}, v\right)=a_{i}\left(e_{D}^{\{i / p\}}, v\right)-a_{i}\left(e_{D}^{\{(i-1) / p\}}, v\right) .
$$

Using 47) with $m=i-1$ with 48 leads to

$$
a_{i}\left(e_{W}^{\{i / p\}}, v\right)=a_{i}\left(e_{D}^{\{i / p\}}, v\right)-\sum_{r=1}^{i-1} a_{i r}\left(e_{W}^{\{r / p\}}, v\right),
$$

thus showing 45. A similar argument shows 466) for $k \geq 1$.

Lemma 4 (Sums of bilinear form with discretization errors with homogeneous bcs). For $0 \leq Q \leq K-1$ we have

$$
\sum_{k=0}^{Q} a_{i}\left(e_{W}^{\{k+i / p\}}, v\right)=a_{i}\left(e_{D}^{\{Q+i / p\}}, v\right)-\sum_{k=0}^{Q} \sum_{r=1}^{i-1} a_{i r}\left(e_{W}^{\{k+r / p\}}, v\right)-\sum_{k=0}^{Q-1} \sum_{r=i+1}^{p} a_{i r}\left(e_{W}^{\{k+r / p\}}, v\right) .
$$

Proof. By Lemma 3 .

$$
\begin{aligned}
\sum_{k=0}^{Q} a_{i}\left(e_{W}^{\{k+i / p\}}, v\right)=\sum_{k=1}^{Q} a_{i}\left(e_{W}^{\{k+i / p\}}, v\right)+a_{i}\left(e_{W}^{\{i / p\}}, v\right) \\
=\sum_{k=1}^{Q}\left\{a_{i}\left(e_{D}^{\{k+i / p\}}, v\right)-a_{i}\left(e_{D}^{\{k-1+i / p\}}, v\right)-\sum_{r=1}^{i-1} a_{i r}\left(e_{W}^{\{k+r / p\}}, v\right)-\sum_{r=i+1}^{p} a_{i r}\left(e_{W}^{\{k-1+r / p\}}, v\right)\right\} \\
\quad+a_{i}\left(e_{D}^{\{i / p\}}, v\right)-\sum_{r=1}^{i-1} a_{i r}\left(e_{W}^{\{r / p\}}, v\right) \\
=\sum_{k=1}^{Q}\left\{a_{i}\left(e_{D}^{\{k+i / p\}}, v\right)-a_{i}\left(e_{D}^{\{k-1+i / p\}}, v\right)\right\}+a_{i}\left(e_{D}^{\{i / p\}}, v\right) \\
\quad-\sum_{k=1}^{Q} \sum_{r=1}^{i-1} a_{i r}\left(e_{W}^{\{k+r / p\}}, v\right)-\sum_{r=1}^{i-1} a_{i r}\left(e_{W}^{\{r / p\}}, v\right)-\sum_{k=1}^{Q} \sum_{r=i+1}^{p} a_{i r}\left(e_{W}^{\{k-1+r / p\}}, v\right) \\
=a_{i}\left(e_{D}^{\{Q+i / p\}}, v\right)-\sum_{k=0}^{Q} \sum_{r=1}^{i-1} a_{i r}\left(e_{W}^{\{k+r / p\}}, v\right)-\sum_{k=0}^{Q-1} \sum_{r=i+1}^{p} a_{i r}\left(e_{W}^{\{k+r / p\}}, v\right) .
\end{aligned}
$$

Lemma 5 (Sum of RHS of the adjoint equations over iterations). Let $2 \leq M \leq p+1$ and $R=M-1$ and $0 \leq Q<K$. Then

$$
\begin{aligned}
& \sum_{k=0}^{Q} \tau_{R}^{Q}\left(e_{W}^{\{k+R / p\}}\right)-\sum_{k=0}^{Q} \sum_{j=M}^{p} a_{R j}\left(e_{W}^{\{k+R / p\}}, \phi^{[Q+j / p]}\right) \\
& =a_{R}\left(e_{D}^{\{Q+R / p\}}, \phi^{[Q+R / p]}\right)-\sum_{k=0}^{Q} \sum_{i=1}^{R-1} a_{i R}\left(e_{W}^{\{k+i / p\}}, \phi^{[Q+R / p]}\right)-\sum_{k=0}^{Q-1} \sum_{i=M}^{p} a_{i R}\left(e_{W}^{\{k+i / p\}}, \phi^{[Q+R / p]}\right) .
\end{aligned}
$$

Proof. From the adjoint equation (21) we have

$$
a_{R}\left(e_{W}^{\{k+R / p\}}, \phi^{[Q+R / p]}\right)=\tau_{i}^{Q}\left(e_{W}^{\{k+R / p\}}\right)-\sum_{j=M}^{p} a_{R i}\left(e_{W}^{\{k+R / p\}}, \phi^{[Q+j / p]}\right) .
$$

From Lemma 4

$$
\begin{aligned}
& \sum_{k=0}^{Q} a_{R}\left(e_{W}^{\{k+R / p\}}, \phi^{[Q+R / p]}\right) \\
& =a_{R}\left(e_{D}^{\{Q+R / p\}}, \phi^{[Q+R / p]}\right)-\sum_{k=0}^{Q} \sum_{i=1}^{R-1} a_{i R}\left(e_{W}^{\{k+i / p\}}, \phi^{[Q+R / p]}\right)-\sum_{k=0}^{Q-1} \sum_{i=M}^{p} a_{i R}\left(e_{W}^{\{k+i / p\}}, \phi^{[Q+R / p]}\right) .
\end{aligned}
$$


Combining (50) and (51) proves the result.

Lemma 6 (Sum of RHS of the adjoint equations over iterations and subdomains). Let $1 \leq M \leq p+1$ and $0 \leq Q<K$. Then,

$$
\begin{aligned}
I=\sum_{k=0}^{Q} \sum_{i=1}^{p} \tau_{i}^{Q}\left(e_{W}^{\{k+i / p\}}\right) & =\sum_{i=M}^{p} a_{i}\left(e_{D}^{\{Q+i / p\}}, \phi^{[Q+i / p]}\right)+\sum_{k=0}^{Q} \sum_{i=1}^{M-1} \tau_{i}^{Q}\left(e_{W}^{\{k+i / p\}}\right) \\
& -\sum_{k=0}^{Q} \sum_{i=1}^{M-1} \sum_{j=M}^{p} a_{i j}\left(e_{W}^{\{k+i / p\}}, \phi^{[Q+j / p]}\right) \\
& -\sum_{k=0}^{Q-1} \sum_{i=M+1}^{p} \sum_{j=M}^{i-1} a_{i j}\left(e_{W}^{\{k+i / p\}}, \phi^{[Q+j / p]}\right) .
\end{aligned}
$$

Proof. The proof is by induction on $M$.

[I] For $M=p+1$ the right-hand side of $(52)$ is simply $I$.

[II] Assume that the expression holds for some $2 \leq M \leq p$.

[III] To show the result is true for $M=p-1$, we isolate terms involving $e_{W}^{\{k+(M-1) / p\}}$.

$$
\begin{aligned}
I & =\sum_{i=M}^{p} a_{i}\left(e_{D}^{\{Q+i / p\}}, \phi^{[Q+i / p]}\right)+\sum_{k=0}^{Q} \sum_{i=1}^{M-2} \tau_{i}^{Q}\left(e_{W}^{\{k+i / p\}}\right)-\sum_{k=0}^{Q} \sum_{i=1}^{M-2} \sum_{j=M}^{p} a_{i j}\left(e_{W}^{\{k+i / p\}}, \phi^{[Q+j / p]}\right) \\
& -\sum_{k=0}^{Q-1} \sum_{i=M+1}^{p} \sum_{j=M}^{i-1} a_{i j}\left(e_{W}^{\{k+i / p\}}, \phi^{[Q+j / p]}\right)+\sum_{k=0}^{Q} \tau_{M-1}^{Q}\left(e_{W}^{\{k+(M-1) / p\}}\right) \\
& -\sum_{k=0}^{Q} \sum_{j=M}^{p} a_{M-1 j}\left(e_{W}^{\{k+(M-1) / p\}}, \phi^{[Q+j / p]}\right) .
\end{aligned}
$$

From Lemma 5

$$
\begin{aligned}
& \sum_{k=0}^{Q} \tau_{M-1}^{Q}\left(e_{W}^{\{k+(M-1) / p\}}\right)-\sum_{k=0}^{Q} \sum_{j=M}^{p} a_{M-1 j}\left(e_{W}^{\{k+(M-1) / p\}}, \phi^{[Q+j / p]}\right) \\
& =a_{M-1}\left(e_{D}^{\{Q+(M-1) / p\}}, \phi^{[Q+(M-1) / p]}\right)-\sum_{k=0}^{Q} \sum_{i=1}^{M-2} a_{i M-1}\left(e_{W}^{\{k+i / p\}}, \phi^{[Q+(M-1) / p]}\right) \\
& -\sum_{k=0}^{Q-1} \sum_{i=M}^{p} a_{i M-1}\left(e_{W}^{\{k+i / p\}}, \phi^{[Q+(M-1) / p]}\right) .
\end{aligned}
$$

Combining (54) with (53),

$$
\begin{aligned}
I & =\sum_{i=M-1}^{p} a_{i}\left(e_{D}^{\{Q+i / p\}}, \phi^{[Q+i / p]}\right)+\sum_{k=0}^{Q} \sum_{i=1}^{M-2} \tau_{i}^{Q}\left(e_{W}^{\{k+i / p\}}\right)-\sum_{k=0}^{Q} \sum_{i=1}^{M-2} \sum_{j=M-1}^{p} a_{i j}\left(e_{W}^{\{k+i / p\}}, \phi^{[Q+j / p]}\right) \\
& -\sum_{k=0}^{Q-1} \sum_{i=M}^{p} \sum_{j=M-1}^{i-1} a_{i j}\left(e_{W}^{\{k+i / p\}}, \phi^{[Q+j / p]}\right) .
\end{aligned}
$$

Corollary 1. Let $0 \leq Q<K$. Then we have

$$
\sum_{k=0}^{Q} \sum_{i=1}^{p} \tau_{i}^{Q}\left(e_{W}^{\{k+i / p\}}\right)=\sum_{i=1}^{p} a_{i}\left(e_{D}^{\{Q+i / p\}}, \phi^{[Q+i / p]}\right)+\sum_{k=0}^{Q-1} \sum_{i=1}^{p} \tau_{i}^{Q-1}\left(e_{W}^{\{k+i / p\}}\right) .
$$


Proof. Set $M=1$ in Lemma 6 to get,

$$
\begin{aligned}
\sum_{k=0}^{Q} \sum_{i=1}^{p} \tau_{i}^{Q}\left(e_{W}^{\{k+i / p\}}\right) & =\sum_{i=1}^{p} a_{i}\left(e_{D}^{\{Q+i / p\}}, \phi^{[Q+i / p]}\right)-\sum_{k=0}^{Q-1} \sum_{i=2}^{p} \sum_{j=1}^{i-1} a_{i j}\left(e_{W}^{\{k+i / p\}}, \phi^{[Q+j / p]}\right) \\
& =\sum_{i=1}^{p} a_{i}\left(e_{D}^{\{Q+i / p\}}, \phi^{[Q+i / p]}\right)-\sum_{k=0}^{Q-1} \sum_{i=2}^{p} \tau_{i}^{Q-1}\left(e_{W}^{\{k+i / p\}}\right) \\
& =\sum_{i=1}^{p} a_{i}\left(e_{D}^{\{Q+i / p\}}, \phi^{[Q+i / p]}\right)-\sum_{k=0}^{Q-1} \sum_{i=1}^{p} \tau_{i}^{Q-1}\left(e_{W}^{\{k+i / p\}}\right),
\end{aligned}
$$

where we use 22, and note that $\tau_{1}^{Q}(v)=0$ for $Q<K-1$.

\subsubsection{Proof of Theorem 3.2 .2}

Proof. From Lemma 2 and 22,

$$
\left(e_{D}^{\{K\}}, \psi\right)=\sum_{k=0}^{K-1} \sum_{i=1}^{p} \sum_{j=1}^{p}\left(e_{W}^{\{k+i / p\}}, \psi_{j}\right)_{i j}=\sum_{k=0}^{K-1} \sum_{i=1}^{p} \tau_{i}^{K-1}\left(e_{W}^{\{k+i / p\}}\right) .
$$

Applying Corollary 1 yields

$$
\left(e^{\{K\}}, \psi\right)=\sum_{i=1}^{p} a_{i}\left(e_{D}^{\{K-1+i / p\}}, \phi^{[K-1+i / p]}\right)-\sum_{k=0}^{K-2} \sum_{i=1}^{p} \tau_{i}^{K-2}\left(e_{W}^{\{k+i / p\}}\right) .
$$

Repeated application of Corollary 1 yields

$$
\left(e^{\{K\}}, \psi\right)=\sum_{k=0}^{K-1} \sum_{i=1}^{p} a_{i}\left(e_{D}^{\{k+i / p\}}, \phi^{[k+i / p]}\right) .
$$

Now,

$$
\begin{aligned}
a_{i}\left(e_{D}^{\{k+i / p\}}, \phi^{[k+i / p]}\right) & =a_{i}\left(u^{\{k+i / p\}}-U^{\{k+i / p\}}, \phi^{[k+i / p]}\right)=a_{i}\left(\widetilde{u}^{\{k+i / p\}}, \phi^{[k+i / p]}\right)-a_{i}\left(\widetilde{U}^{\{k+i / p\}}, \phi^{[k+i / p]}\right) \\
& =l_{i}\left(\phi^{[k+i / p]}\right)-a_{i}\left(\widetilde{U}^{\{k+i / p\}}, \phi^{[k+i / p]}\right)=R_{i}\left(\widetilde{U}^{\{k+i / p\}}, \phi^{[k+i / p]}\right) .
\end{aligned}
$$

Combining (57) and (58) leads to

$$
\left(\psi, u^{\{K\}}-U^{\{K\}}\right)=\sum_{k=0}^{K-1} \sum_{i=1}^{p} R_{i}\left(\widetilde{U}^{\{k+i / p\}}, \phi^{[k+i / p]}\right) .
$$

The discrete equivalent of $(8)$ is

$$
R_{i}\left(\widetilde{U}^{\{k+i / p\}}, v\right)=l_{i}(v)-a_{i}\left(\widetilde{U}^{\{k+i / p\}}, v\right), \quad \forall v \in V_{i, h, 0} .
$$

Substituting $v=\pi_{i} \phi^{[k+i / p]} \in V_{i, h, 0}$ in 60 and subtracting the result from 59 completes the proof.

\subsection{Details of analysis of additive Schwarz algorithm}

\subsubsection{Reformulation of the algorithm}

Similar to the multiplicative case in 86.2 , the basic additive algorithm 2 is not amenable to adjoint based analysis since the affine solution space $H_{D_{k}}^{1}\left(\Omega_{i}\right)$ changes at every iteration. We reformulate the algorithm by again using a standard lifting technique to account for this. We set

$$
\widetilde{u}_{i}^{\{k+1\}}=w_{i}^{\{k+1\}}+u^{\{k\}} \quad \text { on } \Omega_{i}
$$


where now $w_{i}^{\{k+1\}} \in H_{0}^{1}\left(\Omega_{i}\right)$. This results in Algorithm 5 .

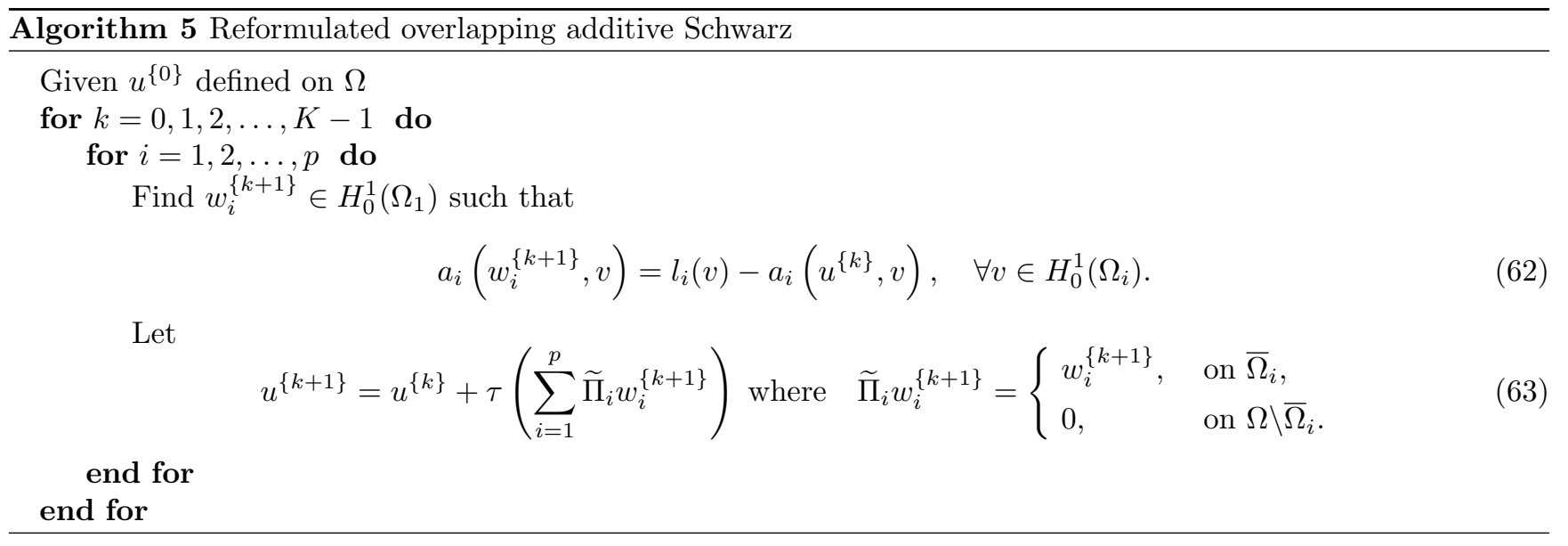

There is an equivalent reformulation of the discrete Algorithm 5 and we denote the unknown solutions as $W^{\{k\}}$ belonging to the spaces $V_{i, h, 0} \subset H_{0}^{1}\left(\Omega_{i}\right)$. These solutions are defined formally but are not computed in practice. Equation (63), which shows that $u^{\{k+1\}}$ is a weighted sum of all previous solutions to (62), results in very different adjoint problems for additive Schwarz (equations (24) from those for multiplicative Schwarz (equations 21) and (22).

To distinguish between different solutions (true, analytical, discrete) we use the notation in Table 15.

\begin{tabular}{||c|c|c|l||}
\hline Notation & Formula & Space & Meaning \\
\hline$u$ & & $H_{0}^{1}(\Omega)$ & True solution \\
$u^{\{k\}}$ & & $H_{0}^{1}\left(\Omega_{i}\right)$ & Global analytic solution at iteration $k$ \\
$U^{\{k\}}$ & & $V_{h}$ & Global discrete solution at iteration $k$ \\
\hline$\widetilde{u}_{i}^{\{k\}}$ & & $H_{D_{k}}^{1}\left(\Omega_{i}\right)$ & Analytic solutions on $\Omega_{i}$ at iteration $k$ \\
$\widetilde{U}_{i}^{\{k\}}$ & & $V_{i, h}^{k}$ & Discrete solutions on $\Omega_{i}$ at iteration $k$ \\
\hline$w_{i}^{\{k\}}$ & & $H_{0}^{1}\left(\Omega_{i}\right)$ & Analytic solns on $\Omega_{i}$ with homogen. bcs at iteration $k$ \\
$W^{\{k\}}$ & & $V_{i, h, 0}$ & Discrete solns on $\Omega_{i}$ with homogen. bcs at iteration $k$ \\
\hline$e^{\{k\}}$ & $u-U^{\{k\}}$ & $H_{0}^{1}(\Omega)$ & Total error \\
$e_{I}^{\{k\}}$ & $u-u^{\{k\}}$ & $H_{0}^{1}(\Omega)$ & Global iteration error at iteration $k$ \\
$e_{D}^{\{k\}}$ & $u^{\{k\}}-U^{\{k\}}$ & $H_{0}^{1}(\Omega)$ & Global discretization error at iteration $k$ \\
\hline$e_{W, i}^{\{k\}}$ & $w_{i}^{\{k\}}-W_{i}^{\{k\}}$ & $H_{0}^{1}\left(\Omega_{i}\right)$ & Discretization error on $\Omega_{i}$ with homogen. bcs at it. $k$ \\
\hline
\end{tabular}

Table 15: Additive Schwarz: notation for different solutions and their spaces.

\subsubsection{Technical lemmas}

Let $e_{W}^{\{k\}}=w^{\{k\}}-W^{\{k\}}$. By 63 we have

$$
e_{D}^{\{k\}}=e_{D}^{\{k-1\}}+\tau \sum_{i=1}^{p} \widetilde{\Pi}_{i} e_{W, i}^{\{k\}}
$$

We apply lemma 1 to arrive at

$$
\left(e_{D}^{\{k\}}, \psi\right)=\left(e_{D}^{\{k\}}, \sum_{i=1}^{p} \chi_{i} \psi\right)=\sum_{i=1}^{p}\left(e_{D}^{\{k\}}, \psi_{i}\right)_{i i}
$$

Lemma 7 (Error in QoI in terms of discretization errors with homogeneous bcs). The discretization error in the QoI is

$$
\left(e_{D}^{K}, \psi\right)=\tau \sum_{k=1}^{K} \sum_{i=1}^{p} \sum_{j=1}^{p}\left(e_{W, i}^{\{k\}}, \psi_{j}\right)_{i j} .
$$


Proof. Using (64), we have for a fixed $j$

$$
\left(e_{D}^{\{K\}}, \psi_{j}\right)_{j j}=\left(e_{D}^{\{K-1\}}, \psi_{j}\right)_{j j}+\tau \sum_{i=1}^{p}\left(e_{W, i}^{\{K\}}, \psi_{j}\right)_{i j},
$$

This is a recursive relation involving $\hat{e}^{\{K\}}$. Unrolling the recursion leads to

$$
\left(e_{D}^{\{K\}}, \psi_{j}\right)_{j j}=\tau \sum_{k=1}^{K} \sum_{i=1}^{p}\left(e_{W, i}^{\{k\}}, \psi_{j}\right)_{i j} .
$$

Summing over all $j=1, \ldots, p$ and using 65,

$$
\left(\hat{e}^{\{K\}}, \psi\right)=\tau \sum_{k=1}^{K} \sum_{i=1}^{p} \sum_{j=1}^{p}\left(e_{W, i}^{\{k\}}, \psi_{j}\right)_{i j} . .
$$

Lemma 8 (Bilinear form with global discretization errors). For any $v \in V_{i}$ we have

$$
a_{i}\left(e_{D}^{\{k\}}, v\right)=\tau \sum_{m=1}^{k} \sum_{j=1}^{p} a_{i j}\left(e_{W, j}^{\{m\}}, v\right) .
$$

Proof. By 64), we have

$$
\begin{aligned}
a_{i}\left(e_{D}^{\{k\}}, v\right) & =a_{i}\left(e_{D}^{\{k-1\}}, v\right)+\tau \sum_{j=1}^{p} a_{i j}\left(e_{W, j}^{\{k\}}, v\right) \\
& =a_{i}\left(e_{D}^{\{k-1\}}, v\right)+\tau \sum_{j=1}^{p} a_{i j}\left(e_{W, j}^{\{k\}}, v\right)
\end{aligned}
$$

since $e_{W}$ is the identity on subdomain $j$. This is a recursive relation involving $a_{i}\left(e_{D}^{\{k\}}, v\right)$. Unrolling this recursion and using the fact that $e_{D}^{\{0\}}=0$ proves the result.

Lemma 9 (Bilinear form with local discretization errors with homogeneous bcs).

$$
a_{i}\left(e_{W, i}^{\{k\}}, \phi_{i}^{[k]}\right)=R_{i}\left(\widetilde{U}_{i}^{\{k\}}, \phi_{i}^{[k]}\right)-\tau \sum_{m=1}^{k-1} \sum_{j=1}^{p} a_{i j}\left(e_{W, j}^{\{m\}}, \phi_{i}^{[k]}\right) .
$$

Proof. By definition of $e_{W, i}^{\{k\}}$,

$$
\begin{aligned}
a_{i}\left(e_{W, i}^{\{k\}}, \phi_{i}^{[k]}\right) & =a_{i}\left(w_{i}^{\{k\}}, \phi_{i}^{[k]}\right)-a_{i}\left(W_{i}^{\{k\}}, \phi_{i}^{[k]}\right) \\
& =a_{i}\left(w_{i}^{\{k\}}+u_{i}^{\{k-1\}}, \phi_{i}^{[k]}\right)-a_{i}\left(u_{i}^{\{k-1\}}, \phi_{i}^{[k]}\right)-a_{i}\left(W_{i}^{\{k\}}+U_{i}^{\{k-1\}}, \phi_{i}^{[k]}\right)+a_{i}\left(U_{i}^{\{k-1\}}, \phi_{i}^{[k]}\right) .
\end{aligned}
$$

Using 61 followed by 62 and definition of $e_{D}^{\{k\}}$,

$$
\begin{aligned}
a_{i}\left(e_{W, i}^{\{k\}}, \phi_{i}^{[k]}\right) & =a_{i}\left(\widetilde{u}_{i}^{\{k\}}, \phi_{i}^{[k]}\right)-a_{i}\left(u_{i}^{\{k-1\}}, \phi_{i}^{[k]}\right)-a_{i}\left(\widetilde{U}_{i}^{\{k\}}, \phi_{i}^{[k]}\right)+a_{i}\left(U_{i}^{\{k-1\}}, \phi_{i}^{[k]}\right) \\
& =R_{i}\left(\widetilde{U}_{i}^{\{k\}}, \phi_{i}^{[k]}\right)-a_{i}\left(e_{D}^{\{k-1\}}, \phi_{i}^{[k]}\right) .
\end{aligned}
$$

By Lemma 8 ,

$$
a_{i}\left(e_{W, i}^{\{k\}}, \phi_{i}^{[k]}\right)=R_{i}\left(\widetilde{U}_{i}^{\{k\}}, \phi_{i}^{[k]}\right)-\tau \sum_{m=1}^{k-1} \sum_{j=1}^{p} a_{i j}\left(e_{W, j}^{\{m\}}, \phi_{i}^{[k]}\right)
$$




\subsubsection{Proof of Theorem 4}

Proof. By (24),

$$
\left(\psi, e_{D}^{\{K\}}\right)=\tau \sum_{k=1}^{K} \sum_{i=i}^{p} \sum_{j=1}^{p}\left(\psi_{j}, e_{W, i}^{\{k\}}\right)_{i j}=\sum_{k=1}^{K} \sum_{i=1}^{p}\left\{a_{i}\left(e_{W, i}^{\{k\}}, \phi_{i}^{[k]}\right)+\tau \sum_{j=1}^{p} \sum_{l=k+1}^{K} a_{i j}\left(e_{W, i}^{\{k\}}, \phi_{j}^{[l]}\right\}\right] .
$$

By Lemma 9 .

$$
\left(\psi, e_{D}^{\{K\}}\right)=\sum_{k=1}^{K} \sum_{i=1}^{p}\left\{R_{i}\left(\widetilde{U}_{i}^{\{k\}}, \phi_{i}^{[k]}\right)-\tau \sum_{m=1}^{k-1} \sum_{j=1}^{p} a_{i j}\left(e_{W, j}^{\{m\}}, \phi_{i}^{[k]}\right)+\tau \sum_{j=1}^{p} \sum_{l=k+1}^{K} a_{i j}\left(e_{W, i}^{\{k\}}, \phi_{j}^{[l]}\right)\right\} .
$$

Application of Galerkin orthogonality, similar to its use in the proof in 6.2 .3 leads to

$$
\left(\psi, e_{D}^{\{K\}}\right)=\sum_{k=1}^{K} \sum_{i=1}^{p}\left\{R_{i}\left(\widetilde{U}_{i}^{\{k\}}, \phi_{i}^{[k]}-\pi_{i} \phi_{i}^{[k]}\right)-\tau \sum_{m=1}^{k-1} \sum_{j=1}^{p} a_{i j}\left(e_{W, j}^{\{m\}}, \phi_{i}^{[k]}\right)+\tau \sum_{j=1}^{p} \sum_{l=k+1}^{K} a_{i j}\left(e_{W, i}^{\{k\}}, \phi_{j}^{[l]}\right)\right\} .
$$

The result follows if

$$
\sum_{k=1}^{K} \sum_{j=1}^{p} \sum_{m=1}^{k-1} \sum_{i=1}^{p} a_{i j}\left(e_{W, i}^{\{m\}}, \phi_{j}^{[k]}\right)=\sum_{k=1}^{K} \sum_{i=i}^{p} \sum_{j=1}^{p} \sum_{l=k+1}^{K} a_{i j}\left(e_{W, i}^{\{k\}}, \phi_{j}^{[l]}\right),
$$

where we interchanged the $i$ and $j$ loop indices on the left hand side. This follows if

$$
\sum_{k=1}^{K} \sum_{m=1}^{k-1} a_{i j}\left(e_{W, i}^{\{m\}}, \phi_{j}^{[k]}\right)=\sum_{k=1}^{K} \sum_{l=k+1}^{K} a_{i j}\left(e_{W, i}^{\{k\}}, \phi_{j}^{[l]}\right) .
$$

To see why this is true, let $A$ be a $K \times K$ strictly lower triangular matrix where the non-zero entries are given by $A_{k, m}=a_{i j}\left(e_{W, i}^{\{m\}}, \phi_{j}^{[k]}\right)$ for $m<k$. Then the left hand side of 66 is the sum of the entries of $A$ by first summing each row while the right hand side of (66) is the sum of the entries of $A$ by first summing each column.

\section{Conclusions and future directions}

We develop an adjoint based a posteriori error analysis to evaluate the discretization and iteration errors for a given quantity of interest when solving boundary value problems using overlapping domain decomposition employing either multiplicative or additive Schwarz iteration. The additional expense of formulating and solving the necessary sequence of adjoint problems both recommends and enables a two stage approach to constructing efficient solution strategies. In this approach, a "stage 1" solution is computed on a relatively coarse discretization employing a small number of iterations or small overlap between subdomains. The error in the quantity of interest is determined for the stage 1 solution and the balance of discretization and iteration errors, and the distribution of discretization error between subdomains, is determined. These guide the solution strategy for a more accurate "stage 2" solution in terms of the localized refinement of the finite element mesh and the choices of overlap and number of iterations.

The adjoint based analysis in this article has focused exclusively on linear problems. Adjoint based analysis can be extended to nonlinear problems, see 31, 21. A consideration of nonlinear problems is therefore an obvious and relatively immediate extension of this work.

A more serious extension is to address initial boundary value problems. In combination with earlier work on parallel methods for initial value problems [10, the current analysis should enable the development of an a posteriori analysis for a numerical method that is parallel in both space and time. Such an analysis would again enable an efficient two stage solution approach, using the distribution of various sources of error estimated from an initial coarse solution to inform the discretization choices for a second "production" computation.

\section{Acknowledgments}

J. Chaudhry's work is supported by the NSF-DMS 1720402. S. Tavener's work is supported by NSF-DMS 1720473.

D. Estep's work is supported by NSF-DMS 1720473. 


\section{References}

[1] Todd Arbogast, Donald Estep, Brendan Sheehan, and Simon Tavener. A posteriori error estimates for mixed finite element and finite volume methods for problems coupled through a boundary with nonmatching grids. IMA Journal of Numerical Analysis, 34(4):1625-1653, 2014.

[2] Todd Arbogast, Donald Estep, Brendan Sheehan, and Simon Tavener. A posteriori error estimates for mixed finite element and finite volume methods for parabolic problems coupled through a boundary. SIAM/ASA Journal on Uncertainty Quantification, 3(1):169-198, 2015.

[3] Wolfgang Bangerth and Rolf Rannacher. Adaptive finite element methods for differential equations. Birkhäuser, 2013.

[4] R. Becker and R. Rannacher. An optimal control approach to a posteriori error estimation in finite element methods. Acta Numerica 2001, 10(1):1-102, 2001.

[5] Varis Carey, D Estep, August Johansson, M Larson, and S Tavener. Blockwise adaptivity for time dependent problems based on coarse scale adjoint solutions. SIAM Journal on Scientific Computing, 32(4):2121-2145, 2010.

[6] Varis Carey, D Estep, and S Tavener. A posteriori analysis and adaptive error control for operator decomposition solution of coupled semilinear elliptic systems. International Journal for Numerical Methods in Engineering, 94(9):826-849, 2013.

[7] J. Chaudhry, N. Burch, and D. Estep. Efficient distribution estimation and uncertainty quantification for elliptic problems on domains with stochastic boundaries. SIAM/ASA Journal on Uncertainty Quantification, 6(3):1127-1150, 2018.

[8] J. H. Chaudhry, D. Estep, V. Ginting, and S.J. Tavener. A posteriori analysis for iterative solvers for nonautonomous evolution problems. SIAM/ASA J. Uncertainty Quantification, 3(1):434-459, 2015.

[9] Jehanzeb H Chaudhry. A Posteriori Analysis and Efficient Refinement Strategies for the Poisson-Boltzmann Equation. SIAM Journal on Scientific Computing, 40(4):A2519-A2542, 2018.

[10] Jehanzeb Hameed Chaudhry, Don Estep, Simon Tavener, Varis Carey, and Jeff Sandelin. A posteriori error analysis of two-stage computation methods with application to efficient discretization and the parareal algorithm. SIAM journal on numerical analysis, 54(5):2974-3002, 2016.

[11] J.H. Chaudhry, D. Estep, V. Ginting, J.N. Shadid, and S.J. Tavener. A posteriori error analysis of IMEX multi-step time integration methods for advection-diffusion-reaction equations. Computer Methods in Applied Mechanics and Engineering, 285:730-751, Mar 2015.

[12] J.H. Chaudhry, D. Estep, V. Ginting, and S.J. Tavener. A posteriori analysis of an iterative multi-discretization method for reaction-diffusion systems. Computer Methods in Applied Mechanics and Engineering, 267:1-22, 2013.

[13] J.H. Chaudhry, D. Estep, and M. Gunzburger. Exploration of efficient reduced-order modeling and a posteriori error estimation. International Journal for Numerical Methods in Engineering, 111(2):103-122, 2017.

[14] J.H. Chaudhry, J.N. Shadid, and T. Wildey. A posteriori analysis of an IMEX entropy-viscosity formulation for hyperbolic conservation laws with dissipation. Applied Numerical Mathematics, 135, 2019.

[15] J Collins, D Estep, and S Tavener. A posteriori error estimates for explicit time integration methods. BIT Numerical Mathematics, 2014.

[16] James B Collins, Don Estep, and Simon Tavener. A posteriori error estimation for the Lax-Wendroff finite difference scheme. Journal of Computational and Applied Mathematics, 263:299-311, 2014.

[17] JB Collins, Donald Estep, and Simon Tavener. A posteriori error estimation for a cut cell finite volume method with uncertain interface location. International Journal for Uncertainty Quantification, 5(5), 2015.

[18] Maksymilian Dryja and Olof B. Widlund. An additive variant of the Schwarz alternating method for the case of many subregions. Technical Report 339, also Ultracomputer Note 131, Department of Computer Science, Courant Institute, 1987. 
[19] Kenneth Eriksson, Don Estep, Peter Hansbo, and Claes Johnson. Introduction to adaptive methods for differential equations. Acta numerica, 4:105-158, 1995.

[20] D. Estep. A posteriori error bounds and global error control for approximation of ordinary differential equations. SIAM Journal on Numerical Analysis, pages 1-48, 1995.

[21] D Estep. Error estimates for multiscale operator decomposition for multiphysics models. In Jacob Fish, editor, Multiscale methods: bridging the scales in science and engineering, pages 305-390. Oxford University Press USA, 2009.

[22] D Estep, V Ginting, and S Tavener. A posteriori analysis of a multirate numerical method for ordinary differential equations. Computer Methods in Applied Mechanics and Engineering, 223:10-27, 2012.

[23] D. Estep, M. Holst, and M. Larson. Generalized Green's functions and the effective domain of influence. SIAM Journal on Scientific Computing, 26(4):1314-1339, 2005.

[24] M.B. Giles and E. Süli. Adjoint methods for pdes: a posteriori error analysis and postprocessing by duality. Acta Numerica, 11(1):145-236, 2002.

[25] Paul Houston, Bill Senior, and Endre Süli. hp-Discontinuous Galerkin finite element methods for hyperbolic problems: error analysis and adaptivity. International Journal for Numerical Methods in Fluids, 40(1-2):153$169,2002$.

[26] A Johansson, JH Chaudhry, Varis Carey, D Estep, V Ginting, M Larson, and S Tavener. Adaptive finite element solution of multiscale pde-ode systems. Computer Methods in Applied Mechanics and Engineering, 287:150-171, 2015.

[27] David E. Keyes, Youcef Saad, and Donald G. Truhlar, editors. Domain-Based Parallelism and Problem Decomposition Methods in Computational Sciences and Engineering. SIAM, 1995.

[28] Gabriel Kron. A set of principles to interconnect the solutions of physical systems. Journal of Applied Physics, 24(8):965-980, 1953.

[29] P. L. Lions. On the Schwarz alternating method III: a variant for nonoverlapping subdomains. In Third international Symposium on domain decomposition methods for partial differential equations, volume 6, pages 202-223. SIAM: Philadelphia, PA, 1990.

[30] Pierre-Louis Lions. On the Schwarz alternating method. I. SIAM, Philadelphia, PA, 1988.

[31] G. I. Marchuk, V. I. Agoshkov, and V. P. Shutyaev. Adjoint Equations and Perturbation Algorithms in Nonlinear Problems. CRC Press, New York, 1996.

[32] Tarek Poonithara Abraham Mathew. Domain Decomposition Methods for the Numerical Solution of Partial Differential Equations, volume 61 of Lecture Notes in Computational Science and Engineering. Springer Berlin Heidelberg, Berlin, Heidelberg, 2008.

[33] J. S. Przemieniecki. Matrix structural analysis of substructures. AIAA Journal, 1(1):138-147, 1963.

[34] Barry F. Smith, Petter E. Bjørstad, and William Gropp. Domain Decomposition: Parallel Multilevel Methods for Elliptic Partial Differential Equations. Cambridge University Press, 1996.

[35] Andrea Toselli and Olof Widlund. Domain Decomposition Methods - Algorithms and Theory, volume 34 of Springer Series in Computational Mathematics. Springer, 2004.

[36] B. Wohlmuth. Discretization methods and iterative solvers based on domain decomposition. Technical report, Habilitation, Department of Mathematics, Augsburg, 1999. 